\title{
QUANTITATIVE ULTRASOUND ASSESSMENT OF THERMAL THERAPY IN LIVER
}

\author{
BY \\ JEREMY PAUL KEMMERER
}

\section{THESIS}

Submitted in partial fulfillment of the requirements

for the degree of Master of Science in Electrical and Computer Engineering in the Graduate College of the

University of Illinois at Urbana-Champaign, 2011

Urbana, Illinois

Adviser:

Assistant Professor Michael L. Oelze 


\begin{abstract}
Quantitative ultrasound (QUS) is a promising technique for non-invasive tissue characterization. In this work, the potential of QUS to assess thermal therapy in liver tissue was examined. Specifically, the QUS parameters of attenuation slope, effective scatterer size (ESD), effective acoustic concentration (EAC), and envelope statistics parameters $\mu$ and $k$ were evaluated with respect to their ability to discriminate between heated and unheated liver tissue. Two sets of experiments were conducted for this purpose. In the first, a series of freshly excised and bisected rat livers was exposed by placing half of a lobe in a saline bath at $60{ }^{\circ} \mathrm{C}$ for 10 minutes and the other half in $37{ }^{\circ} \mathrm{C}$ saline. Each sample was then scanned using single-element ultrasonic transducers with a nominal center frequency of $20 \mathrm{MHz}$. In the second experiment, a series of fresh rat livers was exposed to high-intensity focused ultrasound (HIFU) to produce a discolored region of permanent damage. These samples were scanned in the area of damage before and after HIFU exposure with a $20 \mathrm{MHz}$ single element transducer. Samples in both experiments were preserved, and histology slides were generated.

In the saline bath experiments, statistically significant differences in attenuation slope, ESD, and EAC were observed between heated and unheated samples, while the changes in $k$ and $\mu$ were not statistically significant. Attenuation slope increased on average from $0.65 \mathrm{~dB} / \mathrm{cm} / \mathrm{MHz}$ in untreated liver to $1.1 \mathrm{~dB} / \mathrm{cm} / \mathrm{MHz}$ in treated liver. Statistically significant differences $(\mathrm{p}<0.05)$ in ESD and EAC were observed using spherical Gaussian and fluid-filled sphere models between unheated and heated sides of every sample examined, with ESD decreasing by $34 \%$ and EAC increasing by $18 \mathrm{~dB}$ with thermal insult. A linear model was also considered, and statistically significant increases in slope were observed for all samples, as well as statistically significant decreases in intercept for 5 of 7 samples. The mean $k$ parameter was found to decrease for 5 of 6 samples, although these changes were not statistically significant. Histology slides revealed a decrease of approximately $2.5 \%$ in cell diameter with heating.

The HIFU experiments resulted in an increase in ESD and decrease in EAC in the region of the lesion as determined by visual inspection of the sample. The increases in ESD were significant in 3 of 5 samples for both spherical Gaussian and fluid-filled sphere form-factor models, while the decreases in EAC were statistically significant for 4 of 5 samples for these same models. Statistically significant differences were observed in spectral slope in 3 of 5 samples and in spectral intercept in 2 of 5 samples. Envelope statistics parameters were found to be insensitive to changes with HIFU exposure. Histology slides revealed no appreciable change in cell size.
\end{abstract}


The results of this work suggest that QUS is capable of assessing HIFU and thermal therapy. ESD and attenuation slope were found to have the most potential to discriminate between unheated and heated liver samples in both saline bath and HIFU experiments. 


\section{Acknowledgments}

This thesis represents the efforts of many individuals. I would first like to acknowledge my adviser, Dr. Michael Oelze, for motivating this exciting and important work, and for guidance and support in every step of my research. I would like to thank Dr. William O’Brien Jr. for his insight and suggestions. I would like to acknowledge Dr. Goutam Ghoshal for helping with the majority of the data collection, and Dr. Rita Miller, Dr. Barbara McFarlin, and Jim Blue for providing samples and general guidance throughout my time in the laboratory.

I would like to thank my family and friends for supporting me in the difficult decision and transition in returning to school. This thesis would not exist without them. Finally, I would like to thank my extraordinarily caring and resilient fiancée, Deborah, for patiently enduring my nights and weekends in the laboratory. 


\section{Table of Contents}

Chapter 1: Introduction.

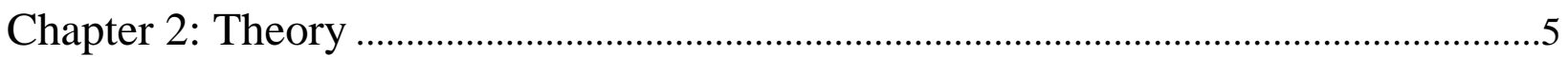

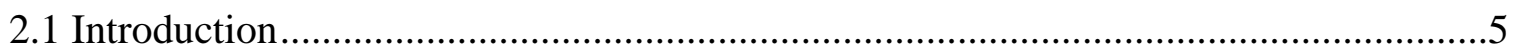

2.2 HIFU Sources and Bio-Heat Transfer...................................................................

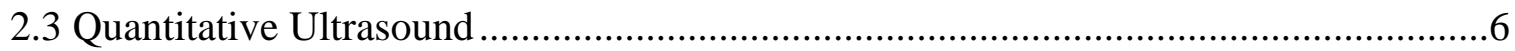

2.3a Backscatter Coefficient and Attenuation Compensation .............................................6

2.3b Effective Scatterer Diameter and Effective Acoustic Concentration ...........................8

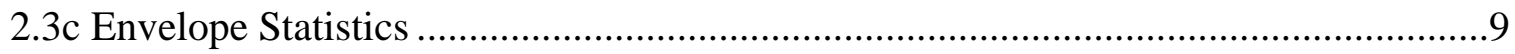

2.3d Attenuation Coefficient...........................................................................................

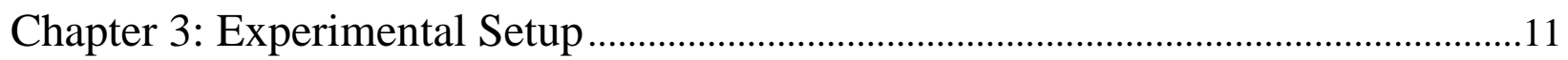

3.1 Introduction .......................................................................................................11

3.2 Sample Preparation Procedures ......................................................................11

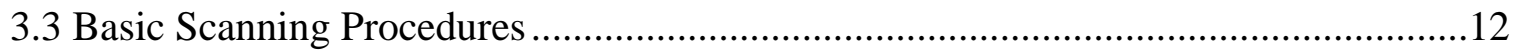

3.4 Saline Bath Experiments........................................................................................14

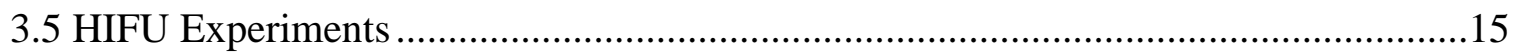

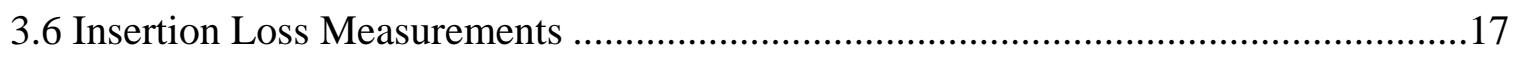

3.7 Histological Analysis ..........................................................................................18

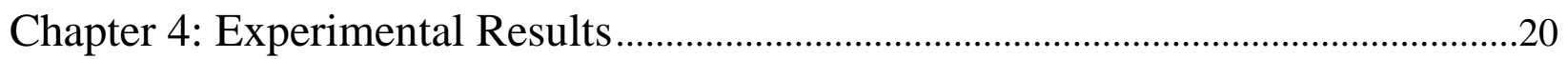

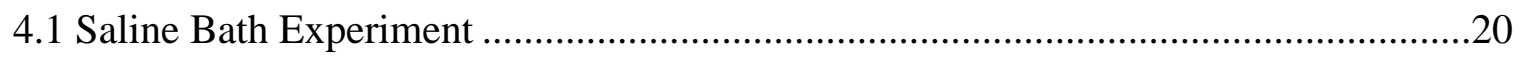

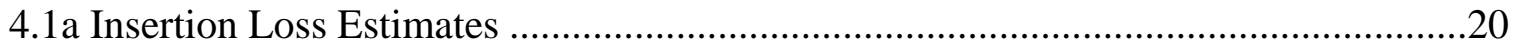

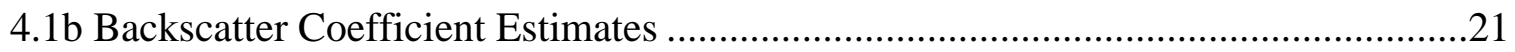

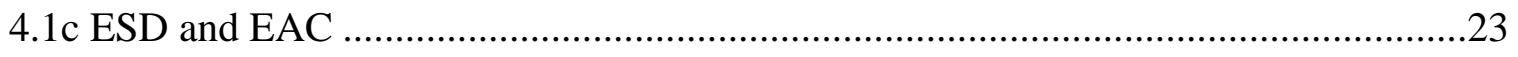

4.1d Envelope Statistics ............................................................................................

4.1e Histological Analysis ........................................................................................

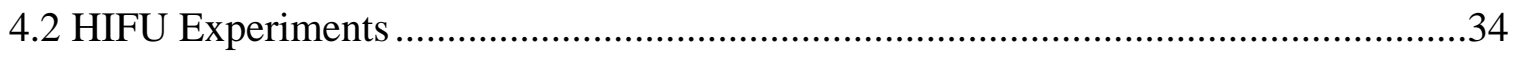




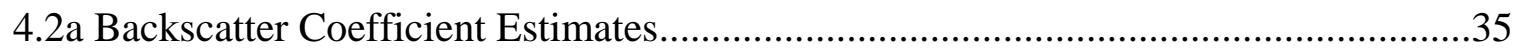

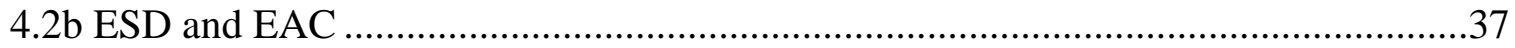

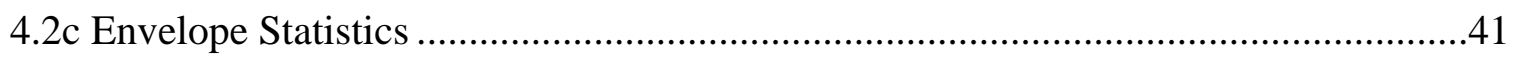

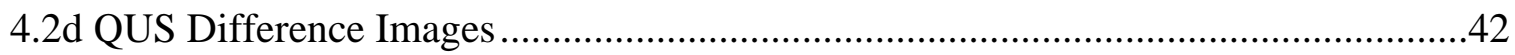

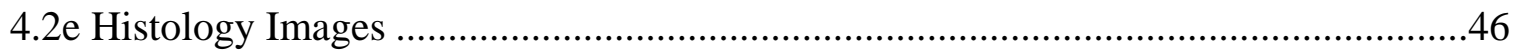

Chapter 5: Conclusions and Future Work ..............................................................................

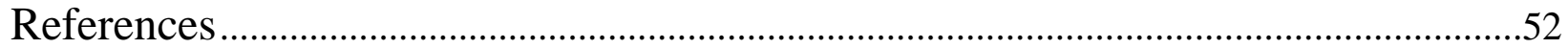




\section{Chapter 1: Introduction}

Diagnostic ultrasound has been in use in the clinic for decades, and yet the full potential of ultrasound to non-invasively image and characterize tissues is still being realized. Diagnostic ultrasound is intrinsically well-suited for the task of medical diagnosis for several reasons. It is inexpensive, portable, safe, and offers high spatial and temporal resolution compared to other imaging modalities in medicine, such as MRI and CT. For these reasons, the number of medically significant areas of investigation using ultrasound continues to grow.

Ultrasound is used not only for diagnostic purposes, but also for many therapeutic applications. High intensity focused ultrasound (HIFU) was first used in neurological research in the 1950s as a means to treat selected parts of the brain while sparing intervening tissues [1]. Since that time, the use of HIFU for therapeutic purposes has been greatly expanded, and novel therapeutic medical applications of ultrasound

are an area of active research. Examples of such therapeutic applications for HIFU are tissue ablation and hyperthermia, with the potential for application in several medical treatments.

Oncology is a particularly important and promising medical application for HIFU, and work in this area has been ongoing for several decades. The goal of HIFU in oncology is the ablation of solid tumors or the application of hyperthermia to solid tumors, usually in combination with another form of treatment. The specialized literature is replete with examples of HIFU for oncological applications [2]. In the 1970s, Fry and Johnson [3] first investigated the destruction of tumors in an animal model using HIFU, and found that survival rate increased for treated animals. Ter Haar et al. [4] demonstrated that liver tumors in a rat model could be destroyed by HIFU without regrowth. Chen et al. [5] demonstrated that continuous destruction of tumor cells was possible with appropriately spaced exposure lesions from HIFU, and later demonstrated [6] complete destruction of fibrosarcoma liver tumors in vivo with an optimized exposure.

While the potential therapeutic value of HIFU has been demonstrated, understanding and controlling HIFU exposure in vivo has proved to be a challenge. The source of damage to cells from HIFU exposure is thought to be a combination of thermal and mechanical mechanisms, with the contribution of each depending on the exposure conditions. Hill [7] developed a framework for predicting HIFU lesion size and shape considering only a thermal mechanism which demonstrated good agreement with experiment. Damianou and Hynynen [8] produced another model using thermal dose as a predictor of lesion volume that also only considered thermal effects. Both the authors acknowledge the potential for a cavitation mechanism for damage, however, and Hynynen [9] concluded that the cavitation threshold in dog muscle was on the order of intensity used for HIFU exposure and could not be excluded from consideration. 
Because of the potential risk of exposure of healthy tissues or uncontrolled cavitation, precise realtime monitoring and control of HIFU is critical. MRI has been used to monitor HIFU-induced temperature changes. McDannold et al. [10] used MRI thermometry for monitoring HIFU application in the brain in a monkey model. Hynenen et al. [11] evaluated MRI thermometry in treating fibroadenoma of the breast with focused ultrasound. Although MRI has been successfully demonstrated for monitoring temperature during focused ultrasound therapy, high equipment cost and instrument compatibility issues limit the availability of MRI as an option for HIFU treatment monitoring.

Ultrasound monitoring of thermal exposure has been investigated actively because of the favorable cost and high temporal frame rate of ultrasound compared to MRI. The use of ultrasonic imaging to monitor HIFU therapy has been explored by several researchers. Bamber and Hill [12] showed that the acoustic attenuation coefficient in tissues is sensitive to temperature. Gertner et al. [13] examined changes in ultrasonic backscatter and attenuation in excised beef liver with temperature, and concluded that ultrasound monitoring of temperature may be feasible. Simon et al. [14] have shown that spatial variations in speed of sound, and therefore temperature, are estimable from backscattered ultrasound, and several authors have developed speed of sound techniques for temperature estimation. Arthur et al. [15] investigated changes in backscattered energy with temperature in hyperthermia. Each of the aforementioned methods has important limitations. The attenuation coefficient is difficult to measure in vivo, although efforts to estimate the attenuation coefficient from backscattered ultrasound are ongoing. Speed of sound approaches suffer from artifacts due to patient motion, which can result in apparent heating artifacts. Techniques that relate the changes in backscattered energy to temperature have only been proposed for hyperthermia applications, and the physical mechanism exploited for temperature estimation is less clear than in other approaches.

Non-invasive assessment of HIFU therapy is also essential for successful therapy application. Treatment feedback is essential for establishing treatment efficacy and for determining if further exposure is required. Bush et al. [16] assessed the viability of using speed of sound, attenuation coefficient, and backscattering coefficient to detect HIFU lesions in pig liver, and concluded that attenuation was the most useful. Zheng and Vaezy [17] concluded that HIFU lesions can be visualized by examining the decay rate of the backscattered RF amplitude of a sample immediately after HIFU exposure. Elastrography techniques [18] have shown potential to differentiate between HIFU lesions and surrounding tissues, as the mechanical properties of the HIFU induced lesions differ from those of untreated tissues. Although the methods to date are encouraging, an ultrasound-based technique which can be readily employed on a clinical device with demonstrated efficacy in vivo is still needed. Because quantitative ultrasound (QUS) 
techniques have been successfully used for tissue characterization, a goal of this work is to investigate how QUS may meet this unsolved challenge of rapid and accurate assessment of HIFU therapy.

Conventional B-mode ultrasound is limited in its potential for detecting disease and assessing therapy because of low contrast between most soft tissues and strong dependence on the skill of the ultrasound technician. QUS refers to a set of techniques which make fuller use of ultrasound data by removing instrument and operator dependency and forming parametric images. These images are hypothesized to quantitatively relate to tissue microstructure and mechanical properties, and may be a new source of tissue contrast. QUS techniques have been used to estimate the scattering properties of the tissue by analyzing the normalized backscattered power spectrum and the statistics of the backscattered signal envelope. From these estimates, spatial maps of estimated properties can be formed to make parametric images, and these QUS parametric images may offer a new source of image contrast for ultrasound, resulting in improved diagnostic capabilities. QUS therefore presents a promising extension of more traditional B-mode imaging in medical applications, with potential applications to the aforementioned problems of therapy monitoring and assessment.

Early work in QUS by Lizzi et al. [19] showed the potential value of analyzing the RF spectrum for diagnostic purposes in the eye and liver. The same group later investigated using spectrum analysis for detecting prostate cancer [20]. Insana et al. [21] provided a theoretical framework for relating the measured power spectrum of a single element transducer to size, number density, and scattering strength of scattering from a random medium such as soft tissue. Oelze et al. [22] investigated using QUS techniques to differentiate between rodent models of breast cancer, and later extended this work to look at higher frequencies [23] in mouse models of breast cancer. These studies concluded that effective scatterer diameter (ESD) and effective acoustic concentration (EAC) could be used to differentiate between different kinds of tumors. Recently, Mamou et al. [24] showed that QUS scatterer size estimates could be used to differentiate between cancerous and cancer-free regions in human patient lymph nodes. The use of envelope statistics combined with spectral features was observed to increase the ability of QUS to detect and classify cancer in rodent models, as well as improve the detection of metastases in the lymph node [25]. Therefore, the use of a larger set of parameters such as spectral features, attenuation, and parameters of the envelope statistics should yield improved diagnostic potential.

QUS research efforts to date include applications for therapy. Lizzi et al. [26] showed that spectrum analysis could provide quantitative information related to changes in tumor tissue microstructure with hyperthermia and tissue ablation. High frequency ultrasound has been demonstrated to be sensitive to cellular changes, such as apoptosis [27] induced by chemotherapy. 
The goal of this work is to explore the feasibility of using QUS techniques to acutely assess areas of damage or lesions in ex vivo tissue caused by simple thermal exposure or by HIFU exposure. This includes differentiating treated tissue from untreated tissue and differentiating tissue damage due to thermal mechanisms from damage due to both thermal mechanisms and possibly cavitation or other mechanical effects. In addition to using QUS to characterize lesions due to thermal damage and possibly cavitation, another goal of this work is to elucidate the structures in thermal lesions giving rise to the QUS parameters.

The next chapters describe the methods and results of experiments designed to quantify changes in scattering in liver tissue with thermal insult. Freshly excised rat liver samples were exposed to thermal insult in a saline bath, and localized heating was generated in rat liver with HIFU. The results of these experiments, as measured by single-element transducer assessment scans, were interpreted with established techniques in quantitative ultrasound and compared to histological slides of the samples used in each experiment. An important goal was to establish a connection between changes in properties of liver tissue estimated with ultrasound and corresponding changes in tissue structure as indicated by histological analysis. 


\section{Chapter 2: Theory}

\subsection{Introduction}

This section includes theory related to predicting thermal increases from HIFU sources and a brief introduction to QUS. In particular, details regarding the estimation of the backscatter coefficient, attenuation coefficient, effective scatter diameter (ESD), effective acoustic concentration (EAC), and envelope statistics are introduced.

\subsection{HIFU Sources and Bio-Heat Transfer}

A thermal mechanism for damage in tissues from focused ultrasound was emphasized in this work. The bio-heat transfer equation was originally proposed by Pennes [28], and describes the temperature field generated by a heat source in tissue:

$$
\dot{T}=k \nabla^{2} T-\frac{T}{\tau}+\frac{q_{v}}{c_{v}}
$$

where $T$ is temperature, $k$ is thermal diffusivity, $\tau$ is the perfusion time constant, $q_{v}$ is the heat source term, and $c_{v}$ is the volume specific heat. When heating is produced ultrasonically, ultrasonic absorption is considered as the source term,

$$
q_{v}=2 \alpha I_{0} \exp (-2 \alpha z)
$$

where $\alpha$ is the absorption coefficient, $I_{0}$ is a spatial and temporal average intensity at the source location, and $z$ is the distance from the surface of the material to source location. Therefore, by increasing the intensity of ultrasound, the rate of change of temperature will also increase for a given level of tissue absorption.

For the case when the source of HIFU is a single-element transducer, as was used in this work, a dynamic spatial variation in temperature results from the spatial variation in field intensity of the source and diffusion of heat according to equation (1). An image of the spatial intensity of a simulated $1-\mathrm{MHz}$ transducer due to the focusing and diffraction of the transducer is shown in figure 1, along with the associated simulated temperature elevation field generated using equations (1) and (2). The size and shape of a HIFU induced lesion will be related to this temperature elevation, which is itself related to the spatial distribution of intensity from the diffraction pattern $[6,7]$. Perfusion is important in in-vivo ultrasonic 
heating, but was not considered in this work. The transducer intensity patterns and temperature maps were used to predict the extent of the heating field produced by the HIFU exposure.
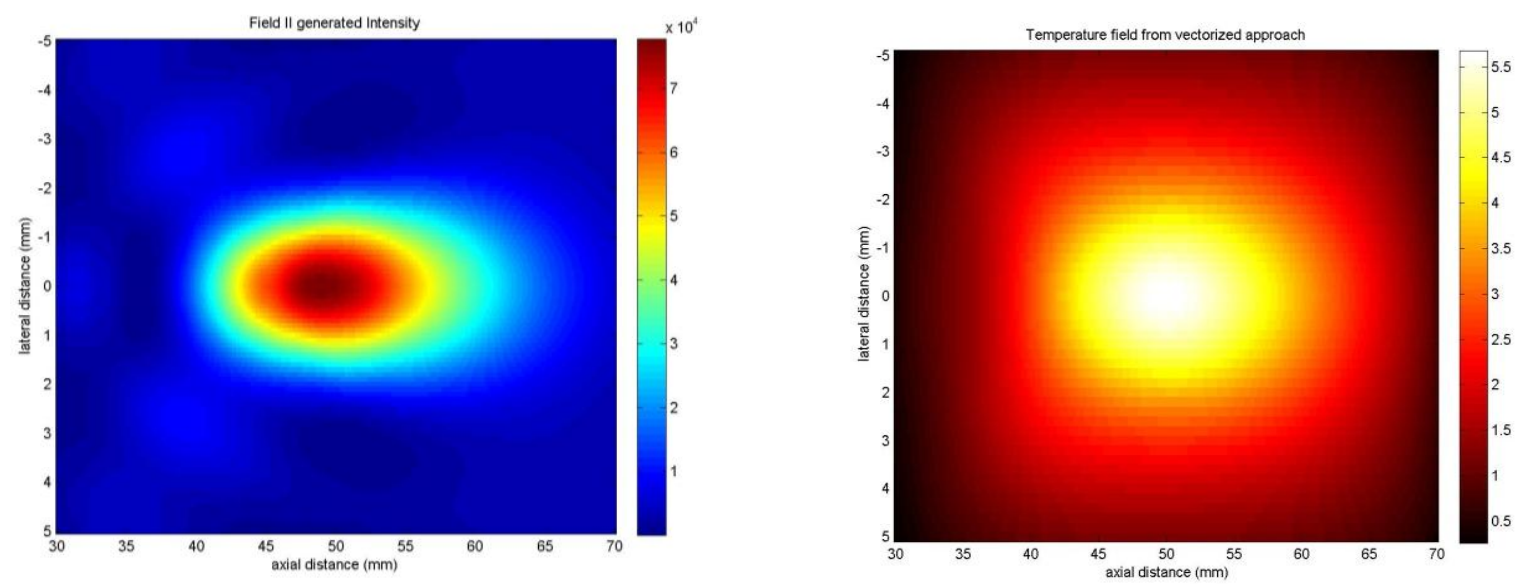

Figure 1 - Simulated intensity (left) and temperature elevation (right) from a 1-MHz source

\subsection{Quantitative Ultrasound}

QUS encompasses several approaches which aim to characterize tissue microstructure or other properties through backscattered ultrasound measurements. This study used two parameters based on the backscatter coefficient (BSC) - the effective scatterer diameter (ESD) and the effective acoustic concentration (EAC) — two parameters derived from the envelope statistics - the $k$ parameter and the $\mu$ parameter - and the ultrasonic attenuation coefficient. In the following subsections, the BSC, ESD, EAC, envelope statistics, and attenuation coefficient are developed and described in terms of backscattered signals.

\section{3a Backscatter Coefficient and Attenuation Compensation}

The BSC is a measure of the backscattered energy of a sample as a function of frequency, and is hypothesized to provide information related to the underlying structure of a sample. The backscatter coefficient is defined as the differential cross section per unit volume evaluated at an angle of $\pi$, which corresponds to the backscatter orientation, and can be represented as:

$$
\sigma_{b}=\frac{|\Phi(k)|^{2}}{V}
$$


where $V$ is the volume of scatterers, and $\Phi(k)$ is the complex scattering amplitude [20].

The BSC can be estimated from backscattered RF data through the normalized power spectrum. The normalized power spectrum for a region of interest (ROI) is calculated from the average of the normalized power spectra from different scan lines in the ROI:

$$
W(k)=\frac{1}{N} \sum_{l=1}^{N}|S(k)|^{2}
$$

where $S(k)$ is the normalized spectrum of a particular scan line, and $N$ is the number of scan line segments in each ROI. In this case, normalization refers to the process of removing instrument dependency from the measurement, and is achieved by compensating with a reference spectrum from a planar reflector or a reference phantom. The signal from the reference will correspond to the same spatial location with respect to the transducer as the ROI in the sample. In the case of using a planar reflector as the reference, the normalized power spectrum is:

$$
|S(k)|=(\gamma / 2) e^{(-2 \alpha d)}\left|\frac{S_{m}(k)}{S_{0}(k)}\right|
$$

where $\gamma$ is the reflection coefficient of the planar reflector, $S_{m}$ is the estimated spectrum from the sample, $S_{0}$ is the measured reference spectrum, $\alpha$ is the difference in attenuation coefficients between the sample medium and the reference medium, and $d$ is the distance to the ROI.

The BSC can be computed as follows:

$$
\sigma_{b} \cong W(k) \frac{R_{1}{ }^{2}}{4\left(0.34 A_{0} z_{c}\right)}
$$

where $W(k)$ is the normalized power spectrum defined in (4), $A_{0}$ is the transducer surface area, $z_{c}$ is the average distance from transducer to ROI, and $R_{1}$ is the on-axis distance between the transducer and the closest surface of the ROI.

The problem of relating frequency-dependant scattering and attenuation to the measured signal can be expressed as: 


$$
\left|\sigma_{b}(f)\right|^{2}=|H(f)|^{2}|S(f)|^{2}|A(f)|^{2}
$$

where $H(f)$ is the system-dependent impulse response and electronic transfer function, and $S(f)$ and $A(f)$ are the frequency-dependant sample scattering function and sample attenuation function, respectively. System dependency can be removed by using a reference measurement, while the scattering and attenuation functions are tissue-dependent and contain useful information for tissue characterization.

\section{3b Effective Scatterer Diameter and Effective Acoustic Concentration}

Two QUS parameters, ESD and EAC, can be estimated from the BSC. The ESD represents a correlation length related to the size of sub-resolution scattering structures. EAC represents the product of the mean square fluctuation in acoustic impedance between a sub-resolution scatterer and the background times the volume fraction of scatterers. ESD is estimated by solving a least-squares problem of comparing BSCs based on measurements with BSCs from theory. For a medium made of many scatterers per resolution cell with random spatial locations, the theoretical BSC can be described through form factors [17]. The form factor is defined as:

$$
F(k)=\sigma_{b} / \sigma_{0}
$$

where $\sigma_{b}$ is the backscatter coefficient of the sample and $\sigma_{o}$ is the backscatter coefficient of a point scatterer. The form factor is a function of the ultrasonic frequency and the size of the scatterers giving rise to the backscattered signal. ESD is designated as the argument minimizing the difference between the log of a model form factor and the log of the form factor derived from measurements [17].

$$
\mathrm{ESD}=\underset{d}{\operatorname{argmin}} \sum_{k=0}^{K}\left[\log \left(\frac{W(k)}{k^{4} F_{m}(k, \mathrm{~d})}\right)-\bar{R}\right]^{2}
$$

where $W(k)$ is the normalized power spectrum, $F_{m}(k, d)$ is the model form factor as a function of size and frequency, and $K$ is the maximum frequency considered. $\bar{R}$ is the average of $W(k) / k^{4} F_{m}(k, d)$ over all frequencies. EAC is then determined as a scaling factor such that the estimated form factor is normalized to unity for $k=0$. 
The spherical Gaussian form factor was used in this work [29], as well as the fluid-filled sphere form factor [30]. The spherical Gaussian form factor represents a scattering particle with a gradual change in impedance compared to the impedance of the background material, and has an effective radius related to the fall-off of the impedance distribution. The spherical Gaussian form factor is defined as:

$$
F_{\text {Gauss }}(2 k)=e^{-0.827 k^{2} a_{e f f}^{2}}
$$

The fluid-filled sphere form factor represents a spherical scattering particle with a clearly defined boundary and surrounded by a uniform medium, and is represented as:

$$
F_{f f s}(2 k)=\left[\frac{3}{2 k a} j_{1}(2 k a)\right]^{2}
$$

where $a$ is the particle radius and $j_{1}$ is the first order spherical Bessel function of the first kind.

\section{3c Envelope Statistics}

Envelope statistics parameters $\mu$ and $k$ were computed for the homodyned $\mathrm{K}$ distribution. The parameter $\mu$ is a measure of the number of scatterers per resolution cell, and the $k$ parameter is the ratio of the coherent to diffuse signal, which can be used to describe the periodicity of a sample. The PDF of the homodyned $\mathrm{K}$ distribution is defined as:

$$
\left.f_{h k}=r \int_{0}^{\infty} x J_{0}(s x) J_{0}(r x)\right)\left(1+\frac{x^{2} \sigma^{2}}{2 \mu}\right)^{-\mu} d x
$$

where $J_{0}$ is the $0^{\text {th }}$ order Bessel function of the first kind, $s^{2}$ is the coherent signal energy, and $\sigma^{2}$ is the diffuse signal energy. The $k$ parameter is computed as $k=s / \sigma$. An efficient method for computing the best-fit parameters for a measured signal envelope was used [31]. 


\section{3d Attenuation Coefficient}

The attenuation coefficient is required for estimation of BSC and QUS parameters derived from BSC. The attenuation coefficient is also valuable for tissue characterization. A common approach for estimating the attenuation of a sample is insertion loss (sec. 3.6). Insertion loss measurements, however, require placement of a known reference reflector in the depth of field of the imaging transducer, which is impractical in most in vivo situations. The attenuation coefficient can be estimated from backscattered ultrasound measurements, and attempts have been made to use these estimates for tissue characterization [32]. In particular, the backscatter-based attenuation estimates have been considered in studying cervical ripening [33]. Results to date, however, suffer from large estimate variance and relatively poor spatial resolution with respect to HIFU lesion size. In this work, only insertion-loss attenuation estimates were considered. 


\section{Chapter 3: Experimental Setup}

\subsection{Introduction}

The experimental data from this work consists of single-element transducer scans of freshly excised rat liver tissue. A portion of each tissue sample was heated either in a saline bath or using an amplified highly focused single-element transducer (HIFU). The data acquisition procedures for these two methods were slightly different, owing to their distinct experimental setup, as described in sections 3.4 and 3.5. Procedures common to both experiments and prerequisite to their execution are found in sections 3.2 and 3.3. Section 3.6 describes the technique of insertion loss, which was used both as a stand-alone parameter for tissue characterization and as part of the later data processing for QUS estimates. Section 3.7 describes the use of histological slides for the evaluation of liver tissue.

\subsection{Sample Preparation Procedures}

Liver samples were excised from Sprague-Dawley rats within 30 minutes of euthanization to avoid sample degradation. These rats were used in other approved studies in the same laboratory, and the experimental protocol was approved by the Institutional Animal Care and Use Committee of the University of Illinois at Urbana-Champaign and satisfied all university and National Institutes of Health rules for the humane use of laboratory animals. Liver samples were cut into lobes, and were either heated in a saline bath by simply setting them on a planar surface or inserted into agar-based phantoms for HIFU exposure.

Agar phantoms containing a single lobe of rat liver were constructed for HIFU experiments. First, $2.5 \mathrm{~g}$ of noble agar was added to $100 \mathrm{~mL}$ of deionized water and stirred using a magnetic stirrer. This agar mixture was then heated in a microwave oven for a total of 60 seconds, removed, and stirred while cooling using a magnetic stir bar. Once the mixture had cooled to approximately $45^{\circ} \mathrm{C}$, a six-well plate was then filled with the agar. Freshly excised lobes of rat liver were then trimmed and added to the agar wells. The well holders containing the liver and agar were stored at $4{ }^{\circ} \mathrm{C}$ for at least 30 minutes to allow the agar to harden. No visible changes in the liver were observed due to embedding in agar. An identical procedure was followed to make agar-only phantoms for calibration and alignment purposes, without the step of adding the tissue sample. 


\subsection{Basic Scanning Procedures}

Single-element transducer scans were performed on all liver tissue samples. Transducers were excited using an Olympus NDT Panametrics 5900 pulser-receiver connected to a 14-bit PC A/D card with $250 \mathrm{MHz}$ sampling. Recorded scan lines were spaced approximately half a beam width apart (125 $\mu \mathrm{m}$ for $20 \mathrm{MHz} / 3$ and $150 \mu \mathrm{m}$ for $20 \mathrm{MHz} \mathrm{f} / 4$ ) and consisted of at least 100 time averages for each scan line. Transducer positioning was achieved using a Daedal positioning system controlled by a PC running custom LabView software. A reference scan was taken for all data sets collected using the same equipment and settings. Reference scans consisted of the measured reflection from a planar Plexiglas reflector at normal incidence. Reference waveforms were obtained with the transducer positioned such that the measurements were taken over the depth of field of the transducer with a step size of $100 \mu \mathrm{m}$ for the 20MHz transducers.

For HIFU exposures, a Sonix RP array system was used for alignment and assessment of HIFU exposures. Data were collected during exposure by synchronizing the Sonix RP array transducer (L14-5) with the excitation signal generator for the HIFU transducer such that the data were collected between periods of HIFU exposure at approximately 30 frames per second. Figure 2 illustrates the timing of exposure and data collection. The measurements continued for several seconds after HIFU exposure ceased. A wire thermocouple (Type T, Omega) was used in several of the measurements to monitor temperature changes. Measurements from a well-characterized reference phantom were taken with the Sonix RP for calibration purposes and using same settings as used in data collection.

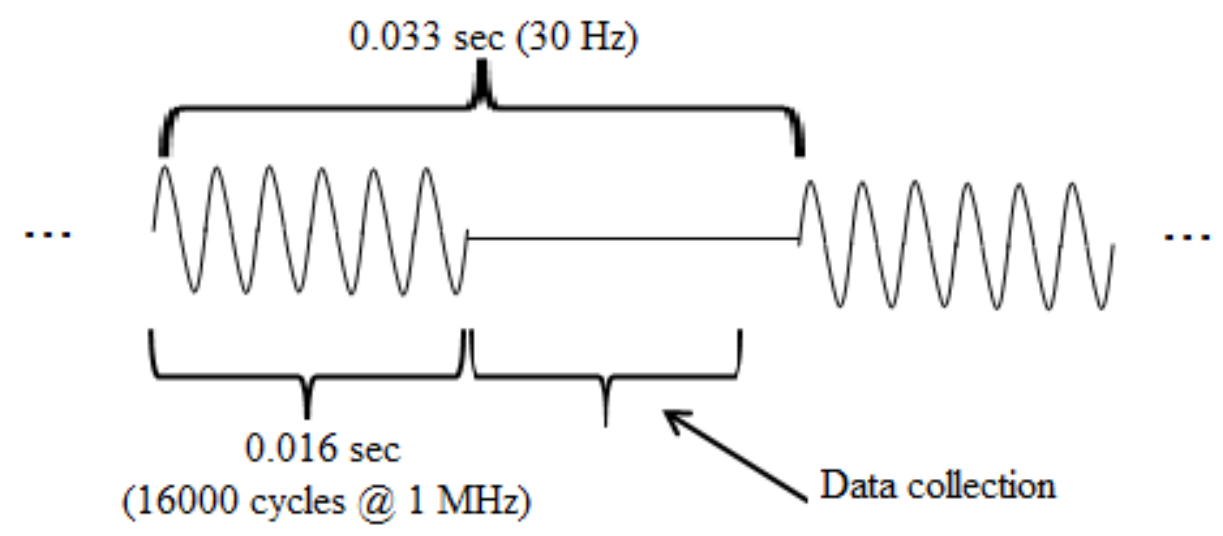

Figure 2 - Timing of HIFU exposure and data collection 
The array transducer (L14-5) was fixed in space with respect to the HIFU transducer such that the field of view of the array overlapped with the HIFU focus. The array probe and single-element HIFU transducer were aligned by creating a single lesion in an agar-only phantom with the HIFU system. This lesion was visible in the display of the Sonix RP system, as the agar melted and left a void, which appeared as an anechoic region on the B-mode display. The array probe was centered laterally on the lesion, and the focus was adjusted axially to match the lesion location. The array transducer remained fixed in space with respect to the HIFU transducer using a clamp holder. The location of the lesion was then marked on the Sonix RP display screen, and subsequent liver samples were placed with respect to the array such that the intended target of the sample appeared at the marked focus on the display. A diagram of this setup is shown in figure 3.

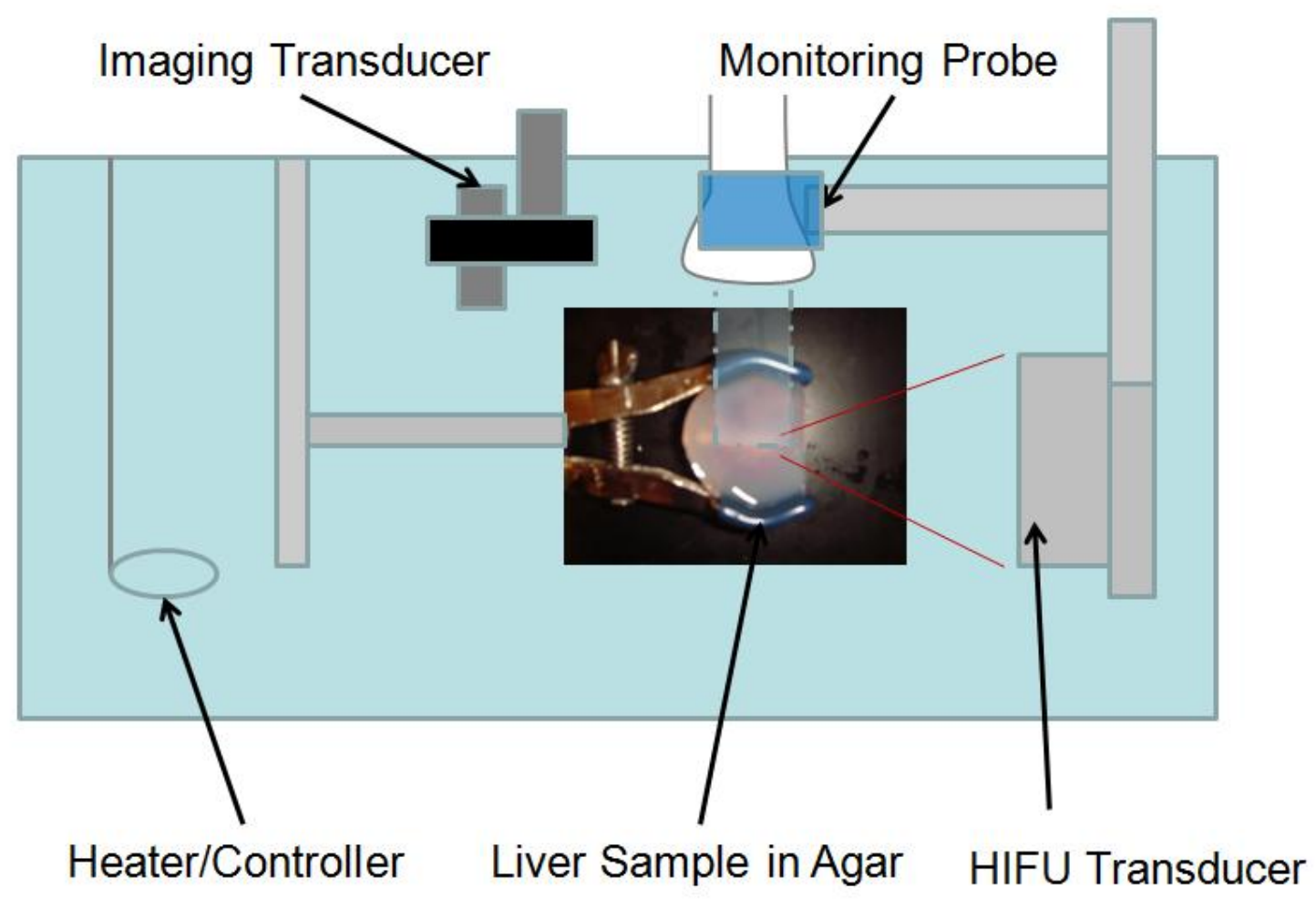

Figure 3 - HIFU exposure experimental setup 


\subsection{Saline Bath Experiments}

HIFU exposure is known to cause damage in tissue by both thermal and mechanical mechanisms, depending on exposure conditions and the state of the tissue. If a tissue has many gas nucleation sites, it is more likely that high intensity ultrasonic fields can cause cavitation resulting in enhanced tissue damage. In order to isolate the effects of thermal damage from mechanical damage due to cavitation, rat liver samples were treated in a saline bath at $60{ }^{\circ} \mathrm{C}$ for 10 minutes. This exposure was chosen to ensure that each sample was uniformly treated. The liver sample was cut in half lengthwise, and half of the sample was treated as described, while the other half was placed in saline at $37^{\circ} \mathrm{C}$ (fig. 4). This treatment regime was chosen to simulate the thermal effects and not mechanical effects of a controlled HIFU exposure. The treatment resulted in a visible change in color and tangible stiffening of the treated liver sample.

The sample halves were pinned to a thin Plexiglas holder and placed in a tank of degassed saline at $37{ }^{\circ} \mathrm{C}$ on top of a Plexiglas reflector (fig. 4). The samples were scanned with a $20-\mathrm{MHz}$ single-element transducer. The scans axes and dimensions were chosen to give several scan slices containing both heated and unheated sections. Figure 5 shows a B-mode image constructed from a saline bath heating experiment data set. The focus of the transducer was positioned to maximize the useful signal, typically placed slightly below the center of the sample in the axial direction. Insertion loss and backscattered RF measurements were obtained from both halves of each sample (sec. 3.3 and 3.6). Histology slides were made from these specimens, as discussed in section 3.7. 


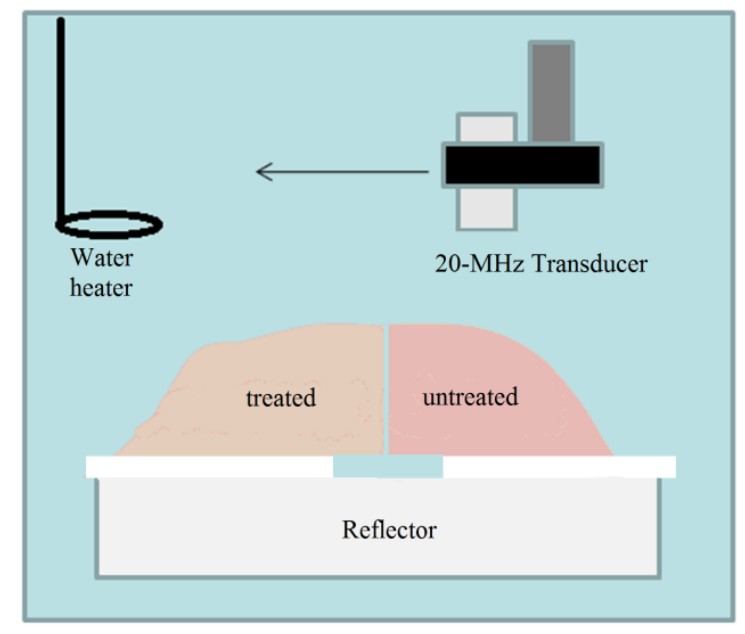

Figure 4 - Saline bath heating experiment

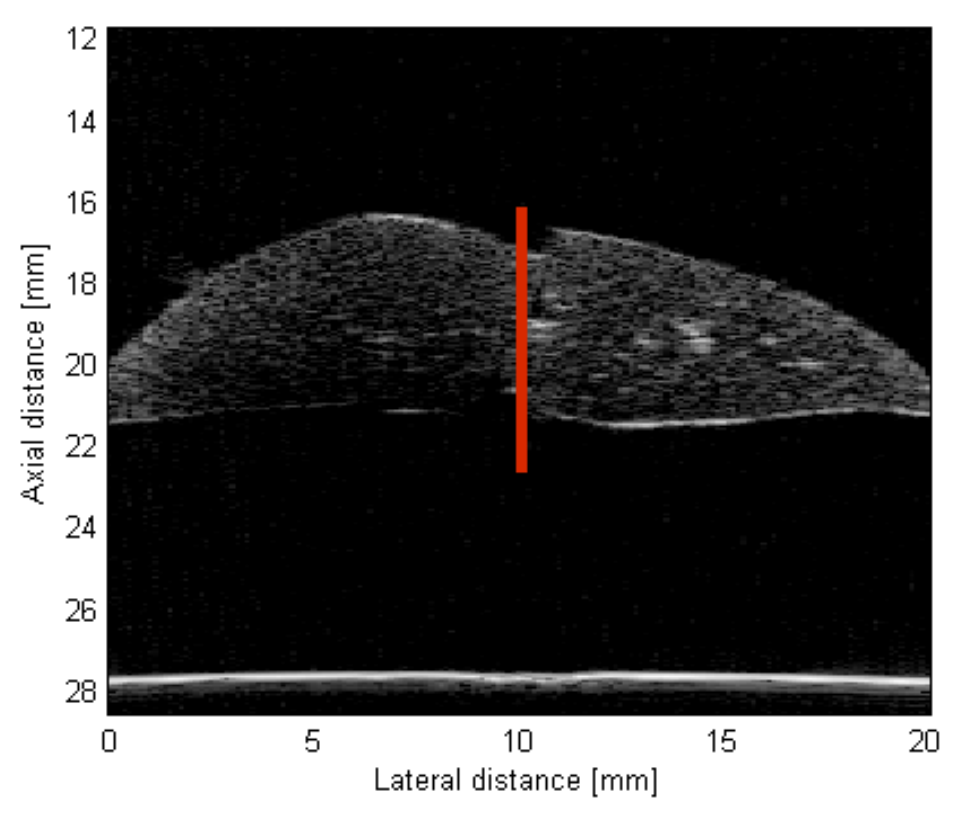

Figure 5 - B-mode image of heated (left) and unheated (right) rat liver sample sections

\subsection{HIFU Experiments}

Liver samples were exposed to a single-element 1-MHz (f/1.1) HIFU transducer to form lesions that were visible upon sample inspection. The HIFU transducer was powered by an ENI A150 $55 \mathrm{~dB}$ power amplifier and excited by an Agilent Technologies 33120a arbitrary waveform generator. Excitation amplitude and duration of the exposure were chosen based on trial and error such that lesions were formed 
which were visible upon sample inspection. Exposure parameters varied from 10-30 seconds with a 50\% duty cycle and peak intensity between 19 and $2500 \mathrm{~W} / \mathrm{cm}^{2}$, as measured in degassed water using a 0.75 $\mathrm{mm}$ Precision Acoustics needle hydrophone (HPM075). The nominal sensitivity of this hydrophone was from $1 \mathrm{kPa}$ to $20 \mathrm{MPa}$ and from $10 \mathrm{kHz}$ to $60 \mathrm{MHz}$. Because of sample attenuation, the in situ intensity was less than the peak intensity measured in water. Lesions were generally formed approximately $1.5 \mathrm{~cm}$ from the front surface of the sample (fig. 6).

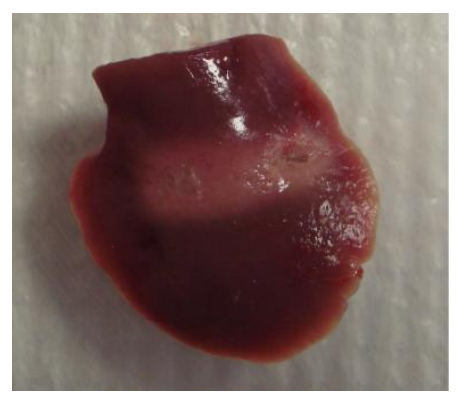

Figure 6 - Photograph of HIFU lesion in rat liver

Ultrasound data were collected during the exposure by synchronizing the RF capture of the Sonix RP probe with quiet periods between HIFU tone-bursts. Increased echogenicity at the focus after HIFU exposure of the liver was sometimes observed in B-mode images from the Sonix RP, and more frequently in the cases of higher intensity and longer exposure.

Liver samples were scanned with a 20-MHz single-element transducer before and after HIFU exposure. Although the samples may have moved slightly with respect to the holder, the before and after scans were taken in the same position with respect to the positioning system, and correlation between the before and after images was generally good based on visible inspection of the various features in the liver (shape, blood vessels, etc). However, HIFU lesions were not generally clearly visible in the B-mode images generated from the liver scans. Liver samples were fixed in 10\% formalin for 2-3 days, and sliced and photographed to establish the location of the lesions within the liver. In this way, the B-mode images were manually registered with photographs of liver slices to select lesion ROIs for data processing (fig. 7). 

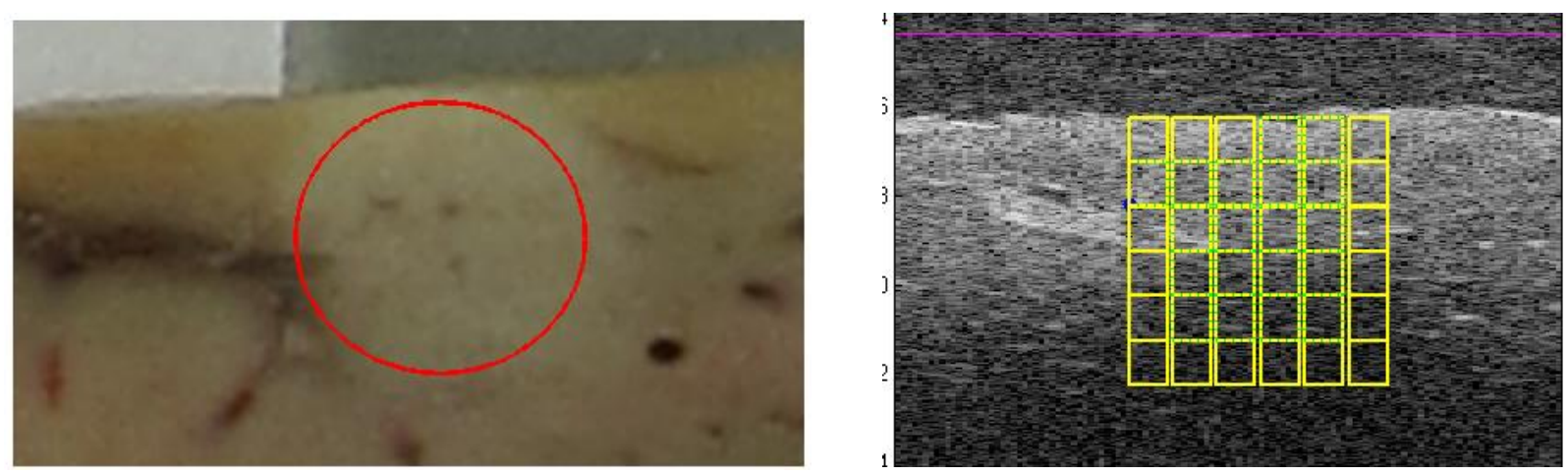

Figure 7 - Sample photograph (left) and B-mode with ROI selection (right)

\subsection{Insertion Loss Measurements}

Insertion loss measurements were performed for liver samples in the saline bath heating experiments in order to estimate changes in attenuation coefficient with heating. A pulse-echo singleelement transducer measurement of the reflection from a planar reflector was obtained with the sample placed between the transducer and the Plexiglas. Several such measurements were acquired over the same lateral region as the backscatter measurements described in section 3.3. A reference measurement was taken in the starting location of this scan with the sample and holder removed, but all other settings maintained. Figure 8 shows reflected signals from the plate with and without the sample between the plate and transducer.

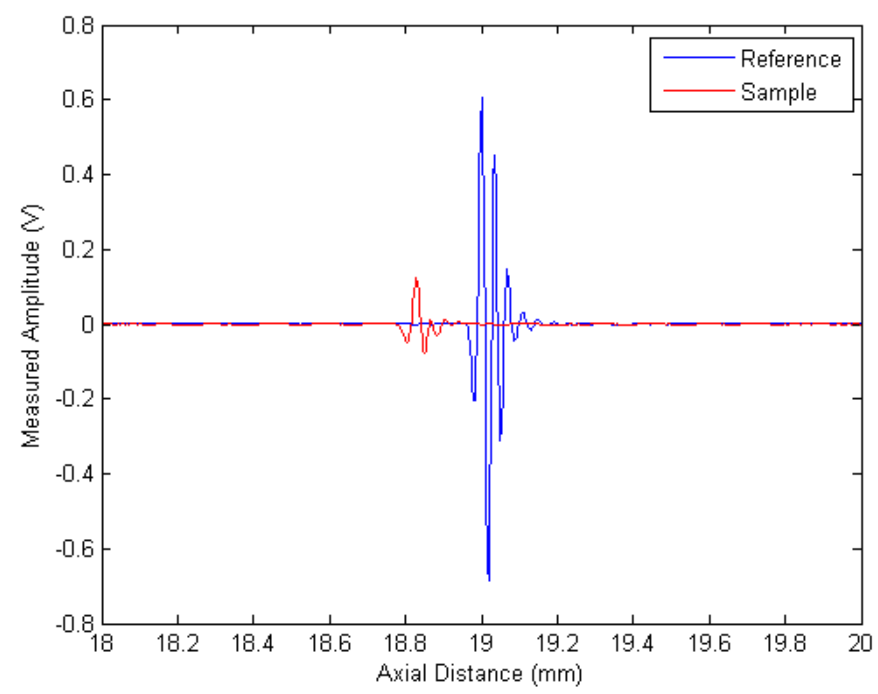

Figure 8 - Sample and reference attenuation measurements 
An insertion loss estimate for the attenuation coefficient $\propto$ from each RF scan line was computed as:

$$
\propto=\frac{10\left[\log _{10}(\operatorname{Pr}(f))-\log _{10}(P s(f))\right]}{2 d f}
$$

where $\operatorname{Pr}(f)$ is the power spectrum of the windowed scan line from the reference measurement, $\operatorname{Ps}(f)$ is the power spectrum with the sample in place, and $d$ is the sample thickness as a function of lateral position. Transmission losses were assumed to be negligible in these measurements.

The sample thickness was estimated ultrasonically using time of flight and the known speed of sound of the saline bath. Specifically, the distances from transducer to sample $(d l)$ and sample to Plexiglas $(d 2)$ were estimated from the measured time corresponding to the interface reflections and the speed of sound in water. The sample thickness was then found as the difference between the total distance from transducer to Plexiglas $(d 3)$ and the distance $d 1+d 2$ as:

$$
t(x, y)=d 3-[d 1(x, y)+d 2(x, y)]
$$

\subsection{Histological Analysis}

Histological analysis is a standard procedure used in pathology to characterize biological samples. In this work, liver samples were preserved by fixation in $10 \%$ formalin for at least 3 days, after which they were stained, mounted in paraffin, and sliced to create microscope slides. The samples were stained using hematoxylin and eosin (H\&E), which are commonly used in medical diagnosis. $\mathrm{H} \& \mathrm{E}$ is a sequence of two agents which stain nuclei blue and protein structures shades of red. Both HIFU and saline bath treated samples were processed for histology, along with corresponding untreated regions and samples in order to determine the extent of structural change induced by HIFU and saline bath heating. A sample histology image is shown in figure 9. 


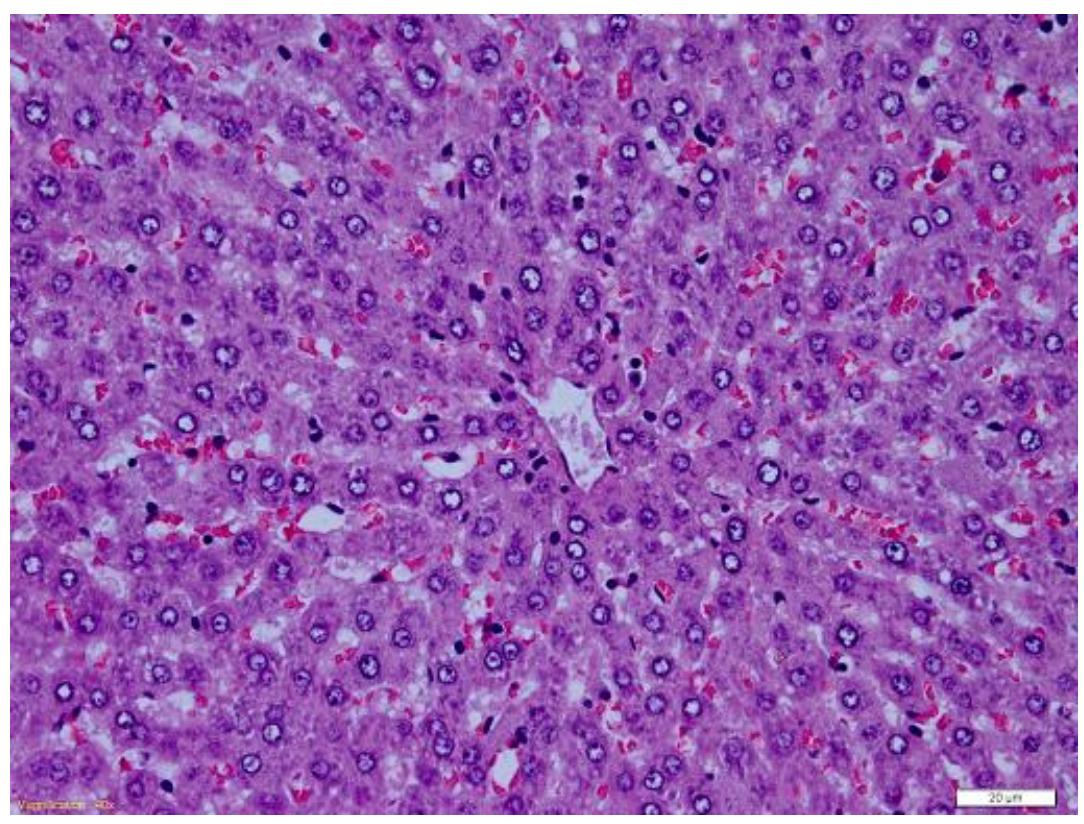

Figure 9 - Histology slide of untreated rat liver sample section 


\section{Chapter 4: Experimental Results}

\subsection{Saline Bath Experiment}

As described in section 3.4, seven rat liver specimens were treated in a saline bath. The samples were bisected, and half were treated at $60{ }^{\circ} \mathrm{C}$ for 10 minutes, with the other half kept at $37{ }^{\circ} \mathrm{C}$. The samples were then scanned using a $20-\mathrm{MHz}$ single-element focused transducer. Insertion loss measurements were performed to obtain accurate estimates of sample attenuation, and estimates of attenuation were found to differ significantly between heated and unheated cases. Co-incident to the scan location of the insertion loss scans, backscattered ultrasound scans were performed over several image slices per sample. The scans encompassed both unheated and heated sections and were used to generate backscatter coefficient, ESD, EAC, and envelope statistics estimates.

\section{1a Insertion Loss Estimates}

Insertion loss measurements revealed substantial increases in attenuation estimates with heating in the saline bath experiment. Specifically, the mean attenuation estimate across all samples for unheated samples was $0.65 \mathrm{~dB} / \mathrm{cm} / \mathrm{MHz}$, while the mean for the heated samples was $1.1 \mathrm{~dB} / \mathrm{cm} / \mathrm{MHz}$, corresponding to an increase of $69 \%$. Figure 10 summarizes these estimates across seven samples and demonstrates a clear separation between heated and unheated cases.

A wide range of values for attenuation in mammalian liver can be found in the literature. Parker [34] found an average attenuation value of $0.4 \mathrm{~dB} / \mathrm{cm} / \mathrm{MHz}$ in bovine liver in the range of 1-6 MHz, while Goss et al. [35] report a value of $0.7 \mathrm{~dB} / \mathrm{cm} / \mathrm{MHz}$ over a similar frequency range while considering a larger number of mammals, including mice. The variation in values of attenuation in liver tissue has been attributed to different measurement techniques, difficulty in measuring sample thickness, and different sample preparation and handling procedures. The estimates in this work fall within the range of values in the literature for unheated samples. Heating complicates the reporting of attenuation values by the addition of experimental variables of exposure time and temperature, and a direct comparison with the literature for the heated case was not attempted. However, substantial increases over unheated values have been reported [13], a fact which agrees with the results estimated in this work. 


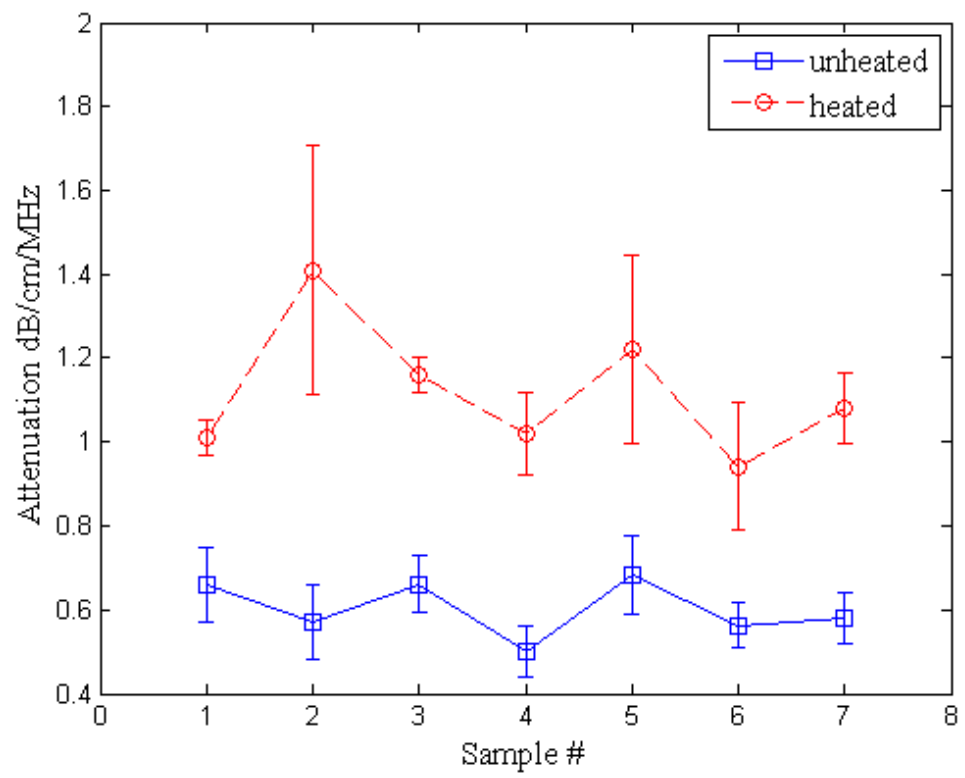

Figure 10 - Attenuation estimates from insertion loss measurements

\section{1b Backscatter Coefficient Estimates}

The BSC provides a measure of the backscattered energy of a sample as a function of frequency, and is valuable as a tool for tissue characterization. In this work, the BSC was estimated from heated and unheated halves of rat liver by computing BSC estimates from independent ROIs. A good agreement across samples and noticeable separation between heated and unheated cases were observed, as shown in figure 11. BSC estimates were calculated assuming an attenuation of $0.65 \mathrm{~dB} / \mathrm{cm} / \mathrm{MHz}$ and 1.1 $\mathrm{dB} / \mathrm{cm} / \mathrm{MHz}$ for unheated and heated regions, respectively, and according to the insertion loss measurements of the same samples (section 4.1a). The BSC curves were then parameterized (i.e., ESD and EAC) according to different scattering models, yielding a geometric interpretation of tissue structural changes occurring due to thermal insult. For example, the larger slopes in the BSCs observed for the heated livers correspond to smaller ESD estimates. 


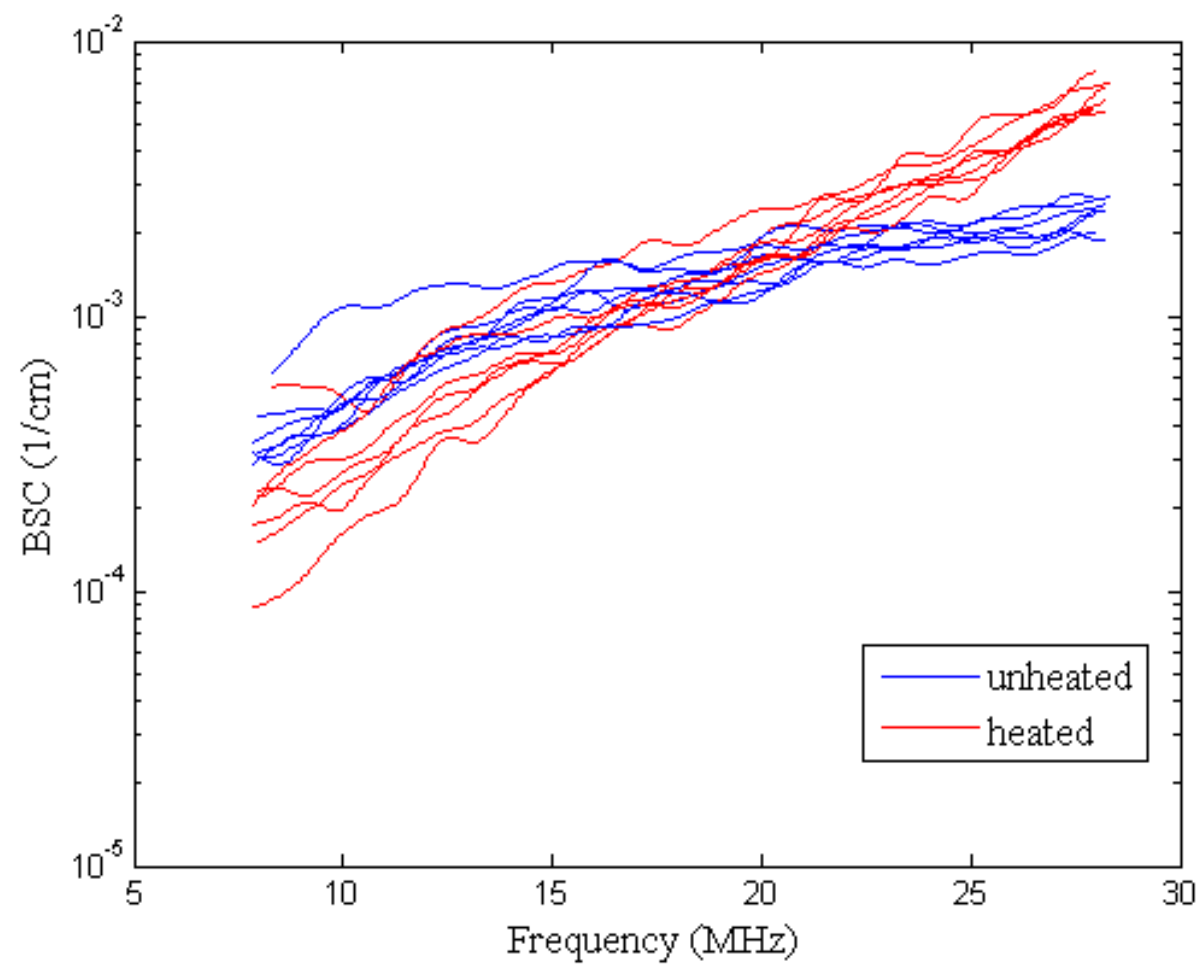

Figure 11 - Backscatter coefficient of heated and unheated livers 


\section{1c ESD and EAC}

ESD and EAC are model-based parameters derived from the frequency-dependent backscattered signal, essentially collapsing the BSC curve into two quantities with a physical interpretation. Figure 12 below shows example QUS parametric images of liver samples from the saline bath experiment using a spherical Gaussian form factor. Differences in estimates of these parameters can be observed in the figures by comparing the heated and unheated cases.
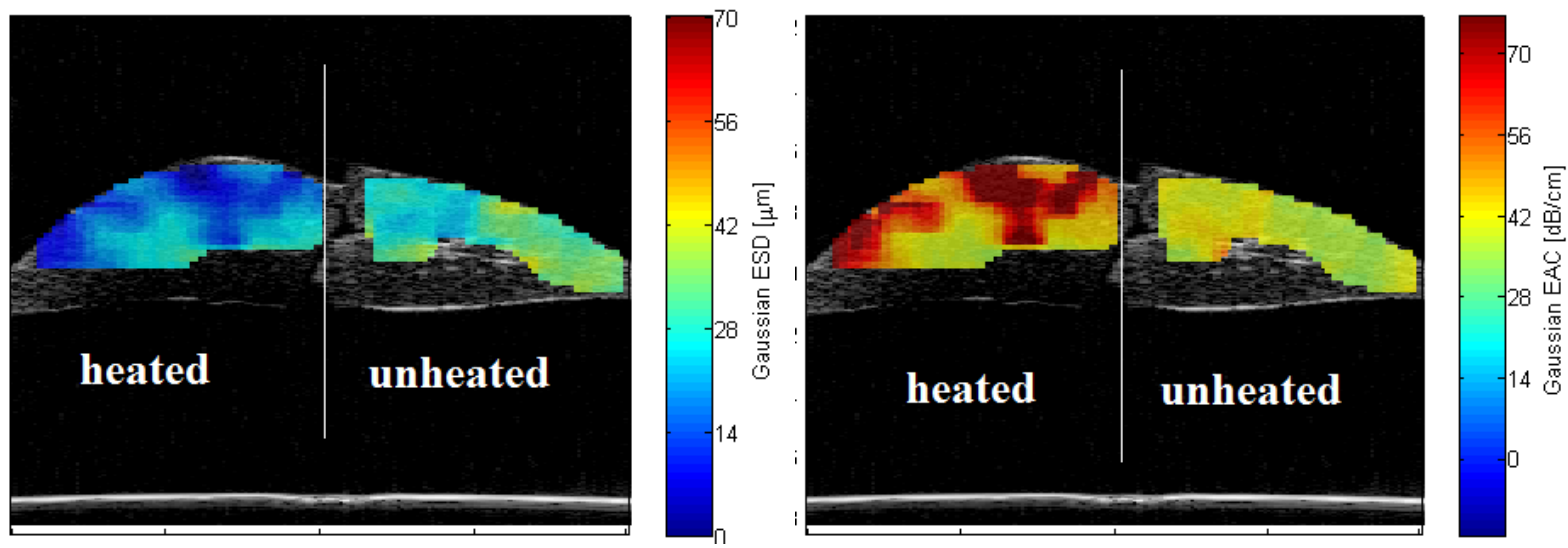

Figure 12 - Parametric images: ESD (left) and EAC (right) (spherical Gaussian form factor)

Data sets were computed from non-overlapping ROIs for statistical analysis, with several image slices per sample. Care was taken to exclude blood vessels or other heterogeneous structures from analysis. Analysis of variance (ANOVA) was performed to test the null hypothesis that the unheated and heated estimates were the same for each liver sample.

The spherical Gaussian and fluid-filled sphere form factors were utilized in this work, along with a linear model parameterized by spectral slope (LS) and spectral intercept (LI). Estimated BSCs versus frequency data were fit using each of these models, and statistics based on these estimated quantities were computed for each sample and model combination. The models were compared in terms of mean-square error (MSE) between the best-fit model and measured data, as well as the statistical significance of differences, if any, between heated and unheated estimate data sets. Figures 13 and 14 show parametric images generated from the same sample image slice for all three of these models. 

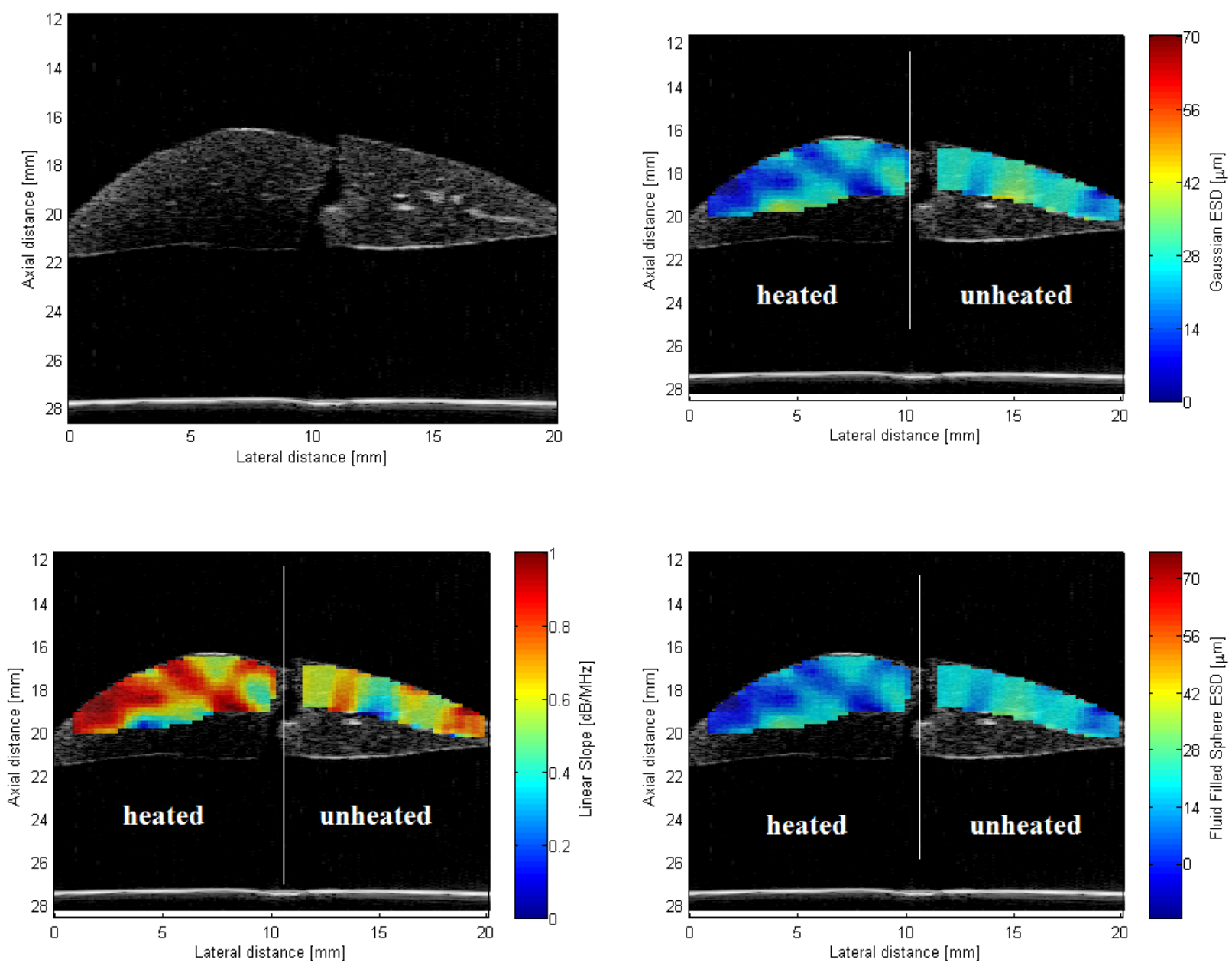

Figure 13 - (Clockwise from top left) B-mode, ESD (Gaussian), LS (linear), and ESD (fluid-filled sphere) images of a rat liver sample 

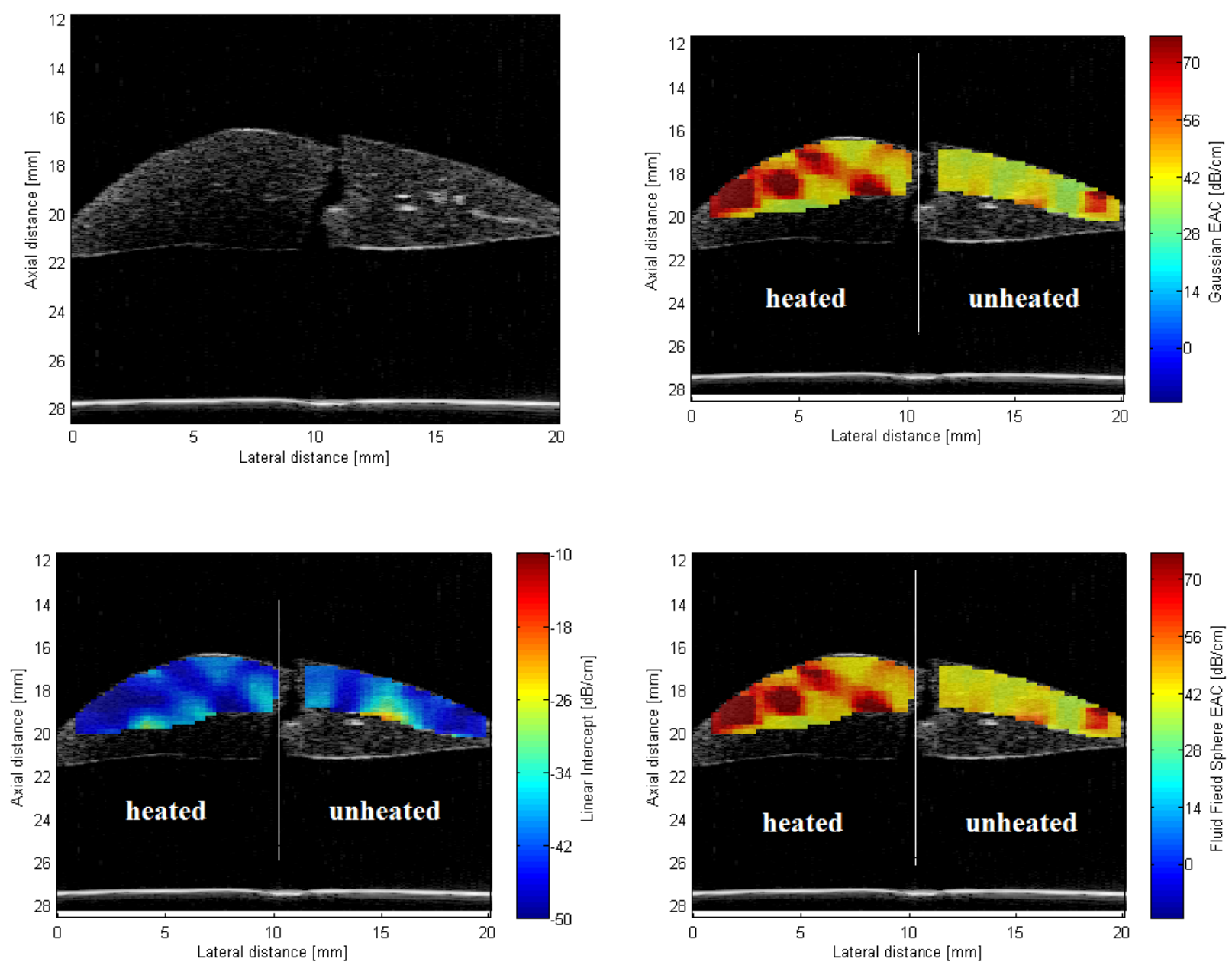

Figure 14 - (Clockwise from top left) B-mode, EAC (Gaussian), LI (linear), and EAC (fluid-filled sphere) images of a rat liver sample

Figure 15 and figure 16 show plots of mean ESD and EAC versus sample number for all samples for the spherical Gaussian and fluid-filled sphere models, respectively, and figure 17 shows LS and LI for the linear model. Unheated ESDs were larger (statistically significant difference, $\mathrm{p}<0.05$ ) than heated ESDs, and unheated EACs were smaller than heated EACs (statistically significant difference, $\mathrm{p}<0.05$ ) for both the spherical Gaussian and fluid-filled sphere form factors. For the linear model, unheated slope estimates were smaller than heated slope estimates at $\mathrm{p}<0.05$ for all samples, and unheated intercept values were larger $(\mathrm{p}<0.05)$ for 5 of 7 samples. The variance was extremely large for heated EACs using spherical Gaussian and fluid-filled sphere models. This can be attributed to the small variations in estimates of size (ESD) leading to very large estimates of EAC. 

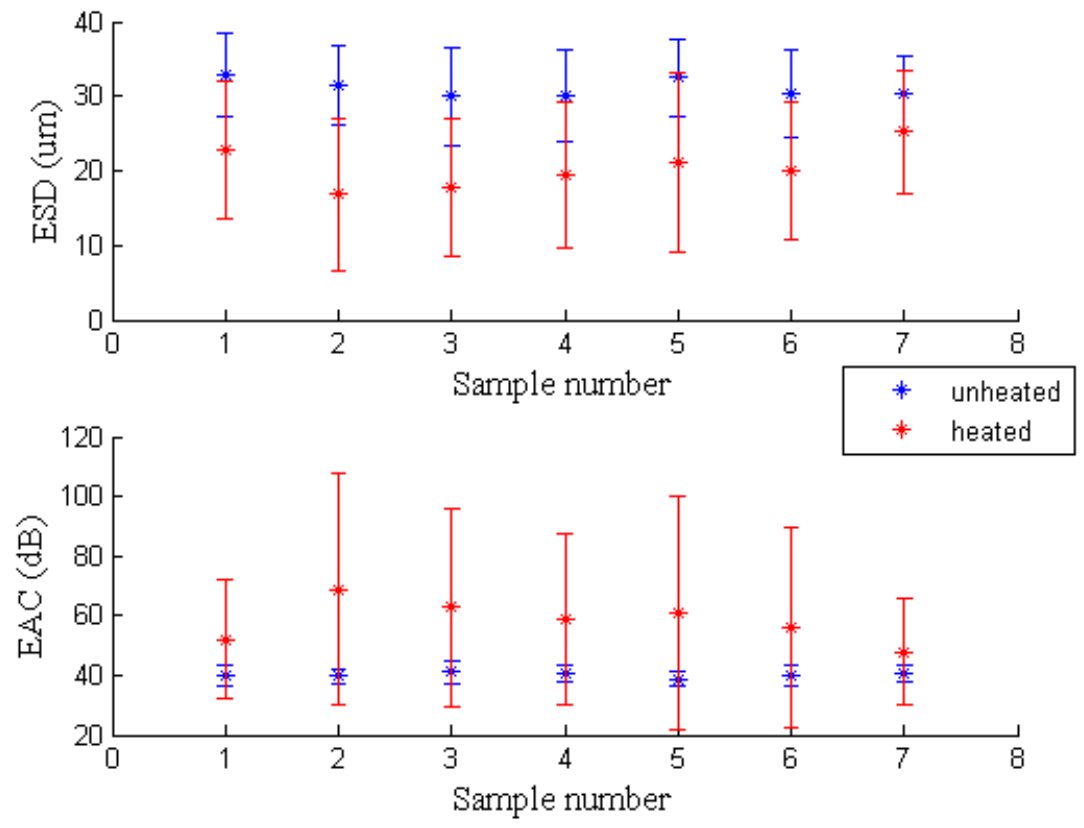

Figure 15 - Mean of ESD and EAC versus sample number (spherical Gaussian form factor)
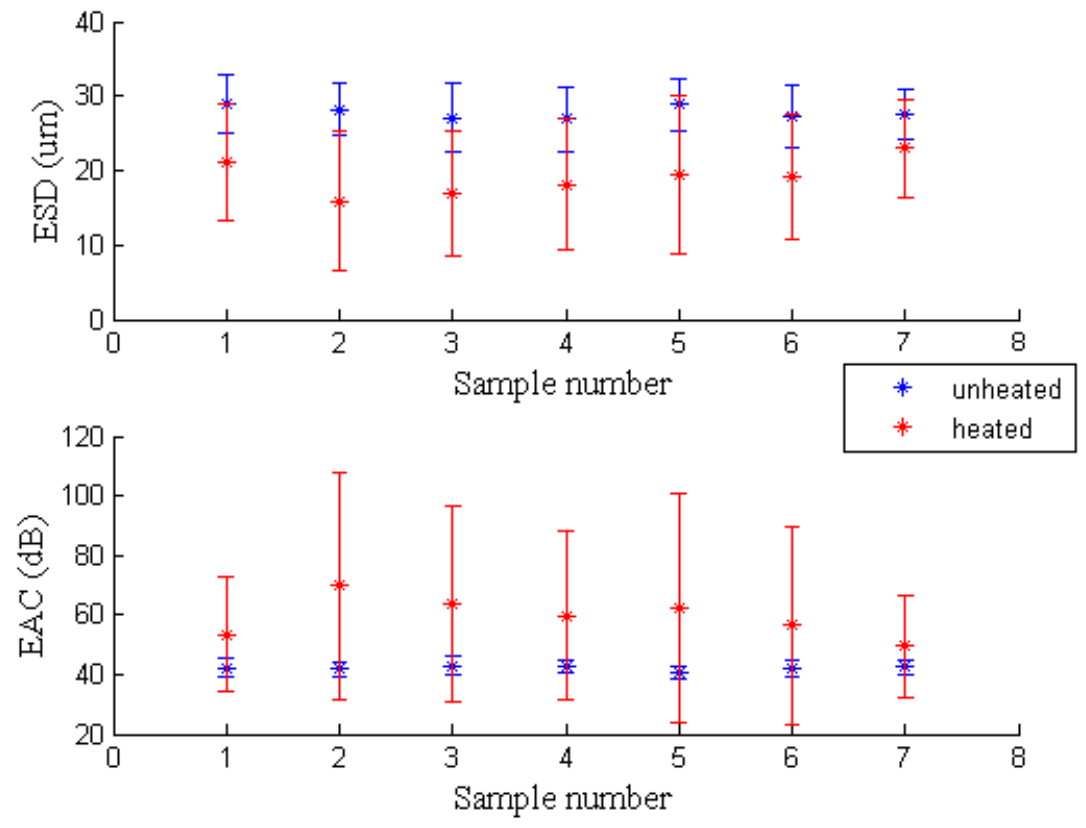

Figure 16 - Mean of ESD and EAC versus sample number (fluid-filled sphere form factor) 


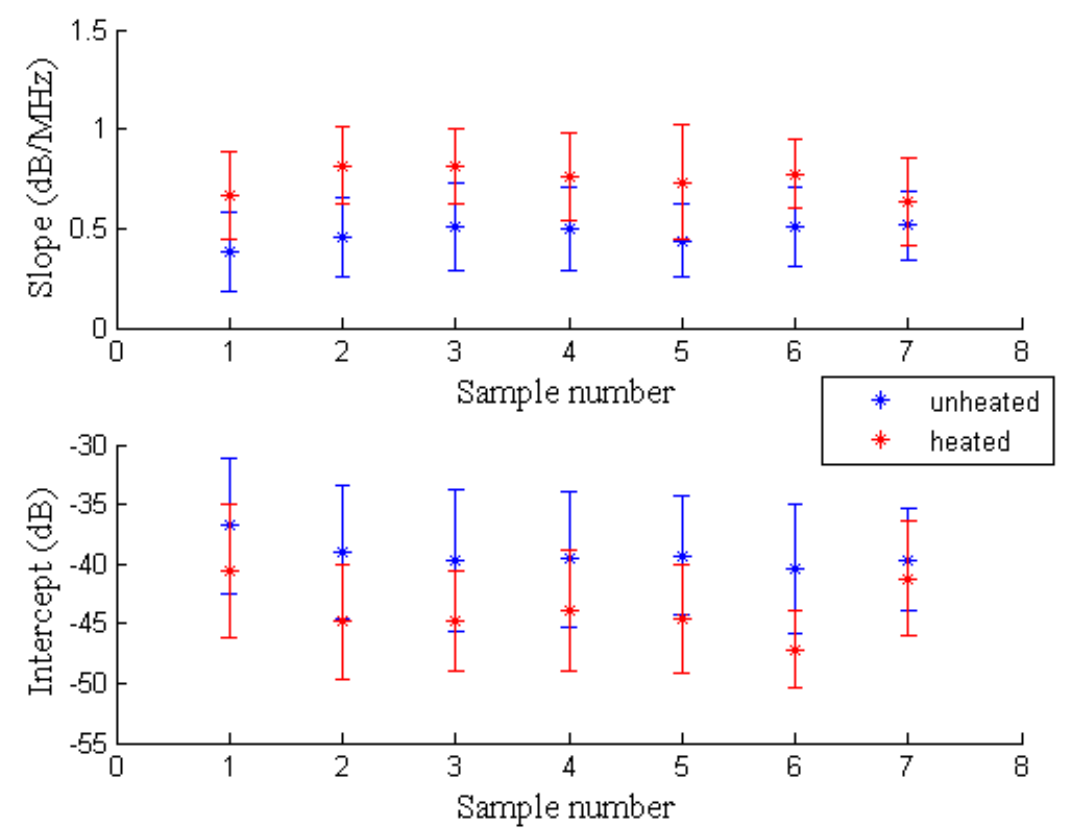

Figure 17 - Mean of ESD and EAC versus sample number (linear model)

Scatter plots of EAC vs. ESD (figs. 18, 19) and LS vs. LI (fig. 20) show separation between heated and unheated samples. The ESD and EAC parameters appeared to be well correlated, suggesting that a single parameter may provide sufficient means to distinguish between heated and unheated samples. Slope and intercept appeared to be correlated for the linear model as well. The scatter plots for the spherical Gaussian and fluid-filled sphere form factors have very similar shape, but size estimates for each model are slightly different. 


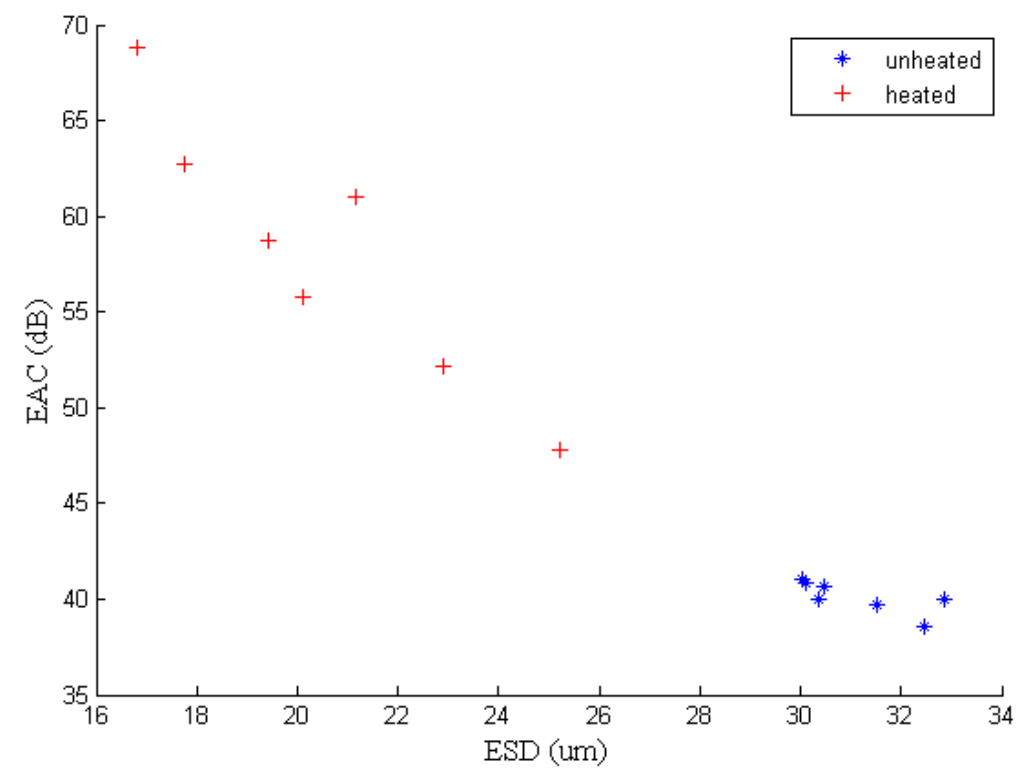

Figure 18 - Scatter plot of liver data estimates (spherical Gaussian form factor)

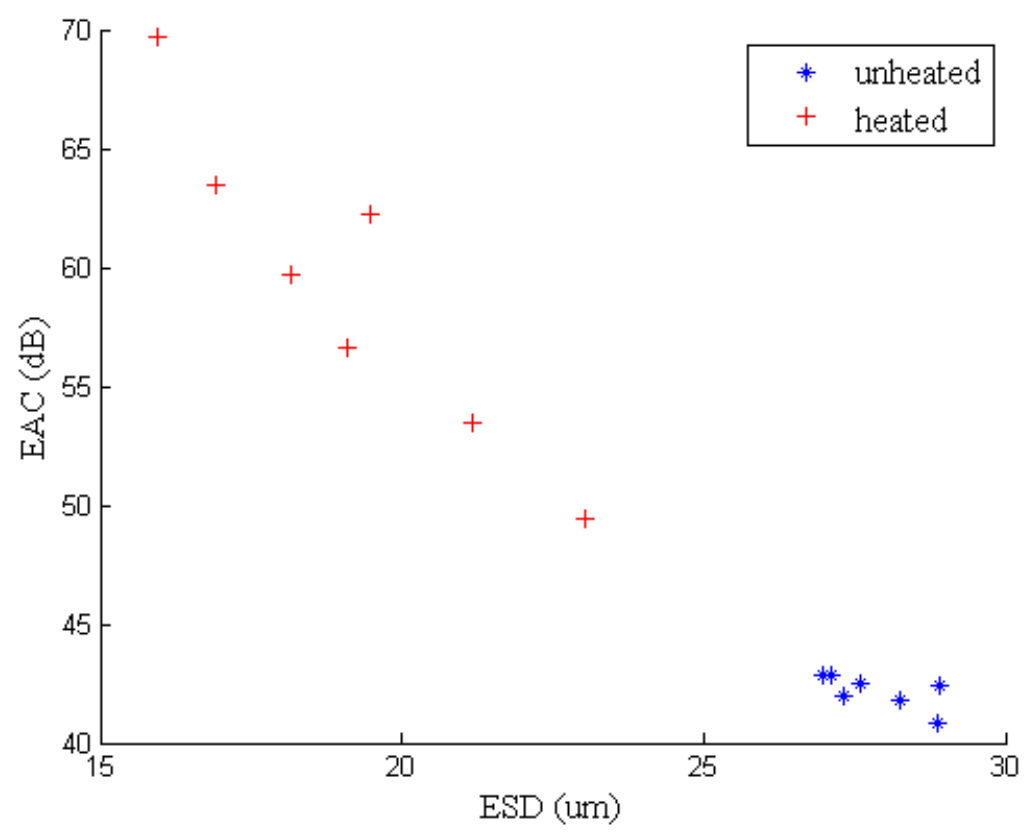

Figure 19 - Scatter plot of liver data estimates (fluid-filled sphere form factor) 


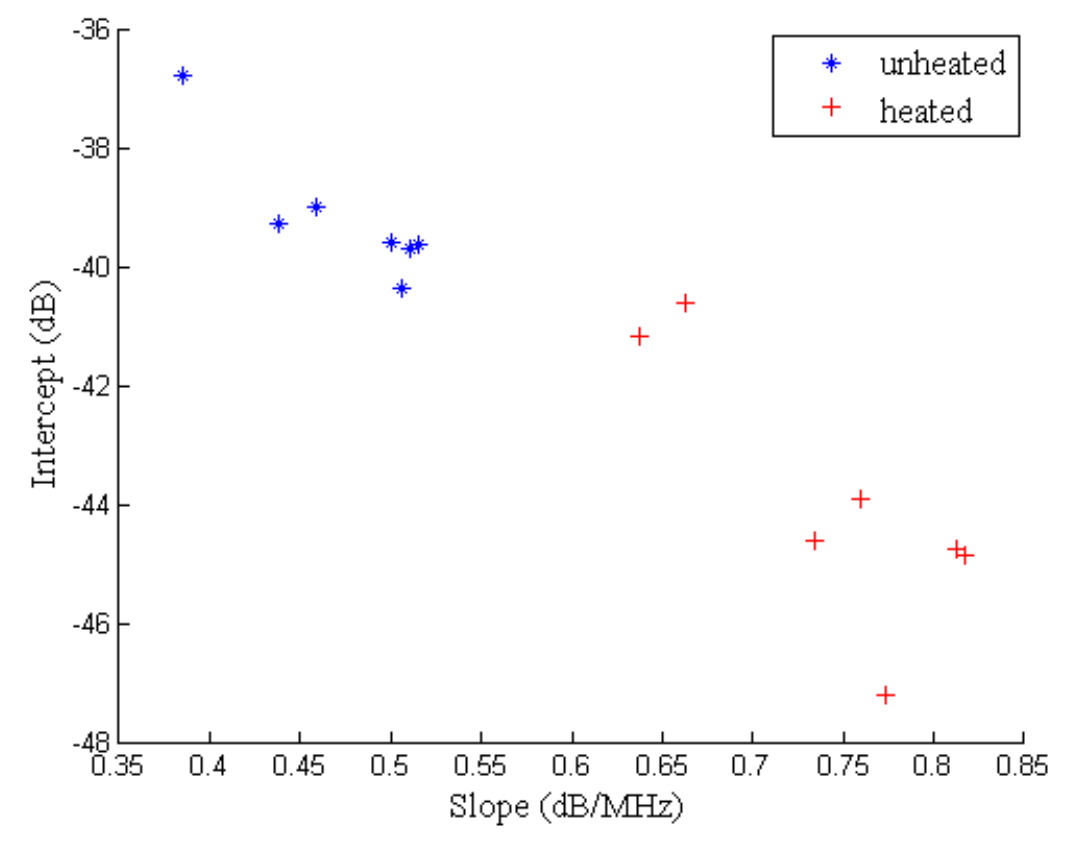

Figure 20 - Scatter plot of liver data estimates (linear model)

Figures 21 and 22 show plots of the average of all BSC measurements along with the best-fit theoretical estimates to the average BSC for each model. Table 1 lists the MSE values for each model compared to measured data. The Gaussian and fluid-filled sphere models produced very similar curves and MSE values, while the linear model had the lowest MSE for both heated and unheated measurement data. 


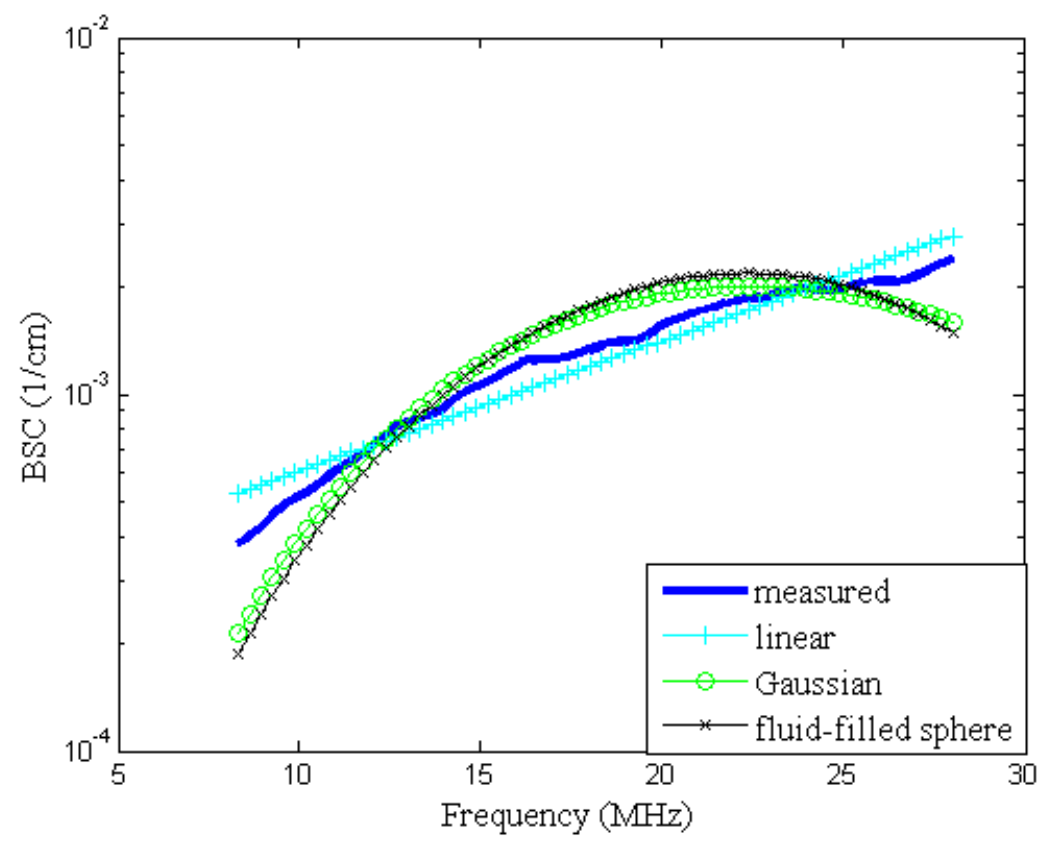

Figure 21 - Measured and modeled BSC (unheated samples)

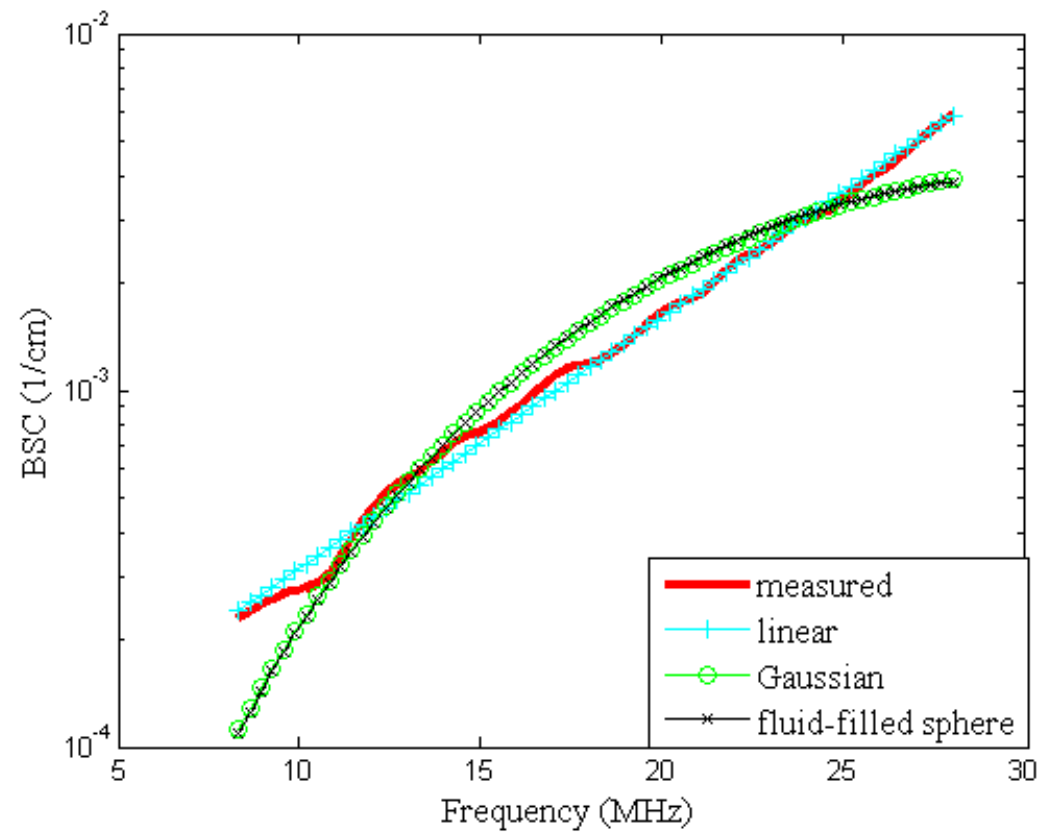

Figure 22 - Measured and modeled BSC (heated samples) 
Table 1 - MSE of measured versus modeled BSC

\begin{tabular}{|c|c|c|}
\hline Form & \multicolumn{2}{|c|}{ Mean-Squared Error (MSE) } \\
\cline { 2 - 3 } Factor & Unheated & Heated \\
\hline Gaussian & 828.7 & 998.5 \\
\hline $\begin{array}{c}\text { Fluid-filled } \\
\text { sphere }\end{array}$ & 1293 & 1085 \\
\hline Linear & 307.9 & 93.80 \\
\hline
\end{tabular}

\section{1d Envelope Statistics}

Envelope statistics parameters $\mu$ and $k$ were computed with the ROIs used for ESD and EAC estimates in section 4.1c. Figure 23 shows a plot of the mean of the envelope statistics parameters, and figure 24 shows a scatter plot of $\mu$ versus $k$. The separation for $\mu$ and $k$ between heated and unheated samples was less pronounced than for ESD and EAC, although some separation in the $k$ parameter was apparent. Although $k$ appears to decrease with heating for most samples, the difference in $k$ between heated and unheated cases was not statistically significant at the $p<0.05$ level for any sample.
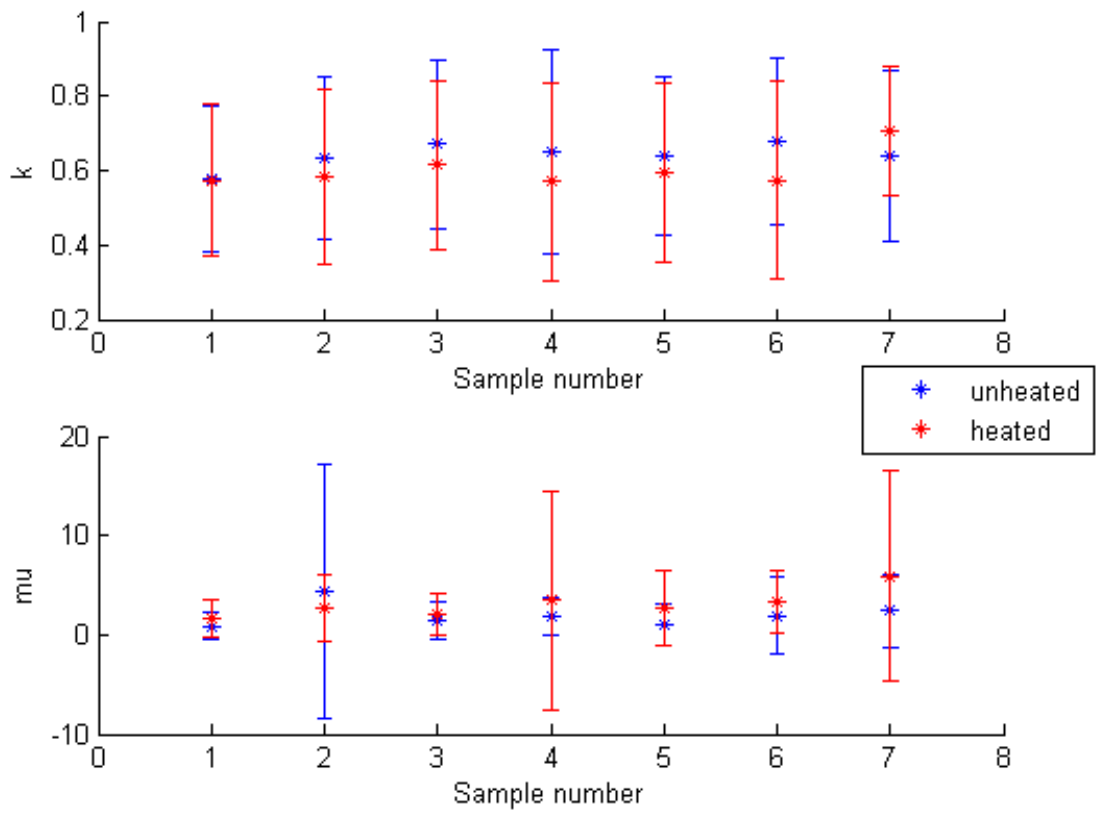

Figure 23 - Mean of envelope statistics parameters 


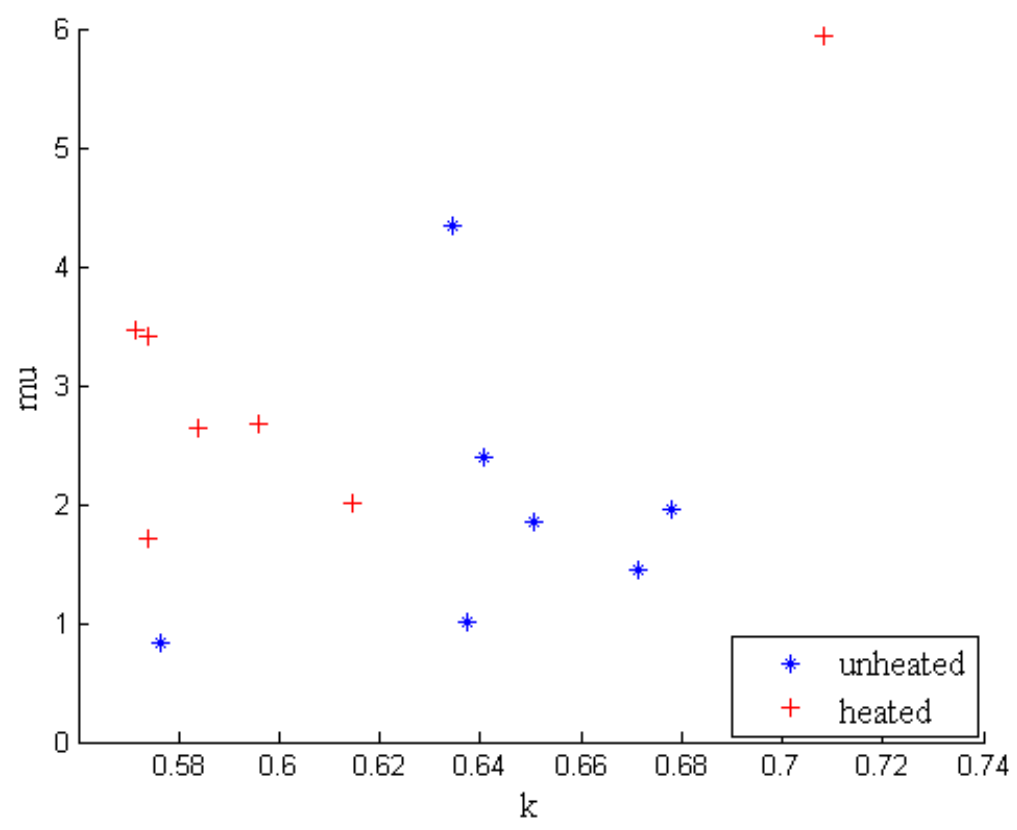

Figure 24 - Scatter plot of envelope statistics parameters

\section{1e Histological Analysis}

Histological slides were created from a saline bath experiment specimen after fixation and $\mathrm{H} \& \mathrm{E}$ staining, as described in section 3.7. Figure 25 shows a digitized slide image from an unheated section of the liver specimen, and figure 26 shows a heated specimen. In the figures, the round, purple structures are cell nuclei. The extent of a single liver cell is more clearly observed in figure 26 (heated), where the spacing between the lighter purple cell cytoplasm appears. These elongated gaps are referred to as sinusoids, and are the capillaries which provide blood to the cells. An apparent shrinking of the liver cells in the heated versus unheated case was observed in regions adjoining the tissue border and around the blood vessels, which was consistent with the ESD estimates derived from measurements from heated and unheated livers, which had smaller ESD values for heated samples. In addition, significant damage to the lining of the larger blood vessels was noted, suggesting that heating in this fashion may affect the tissue blood supply. Figure 27 shows a histogram of estimated cell diameter for heated and unheated sample histology slides. Cells diameter estimates correspond to the largest dimension of a cell, as measured manually from a print of the histology image. The average cell diameter for the unheated cells was $22.7 \pm$ $1.9 \mu \mathrm{m}$, while the average diameter for the heated cells was $22.1 \pm 2.0 \mu \mathrm{m}$. The average cell nucleus diameter was $9.5 \pm 1.0 \mu \mathrm{m}$ for unheated cells, and $9.9 \pm 0.6 \mu \mathrm{m}$ for heated cells. 


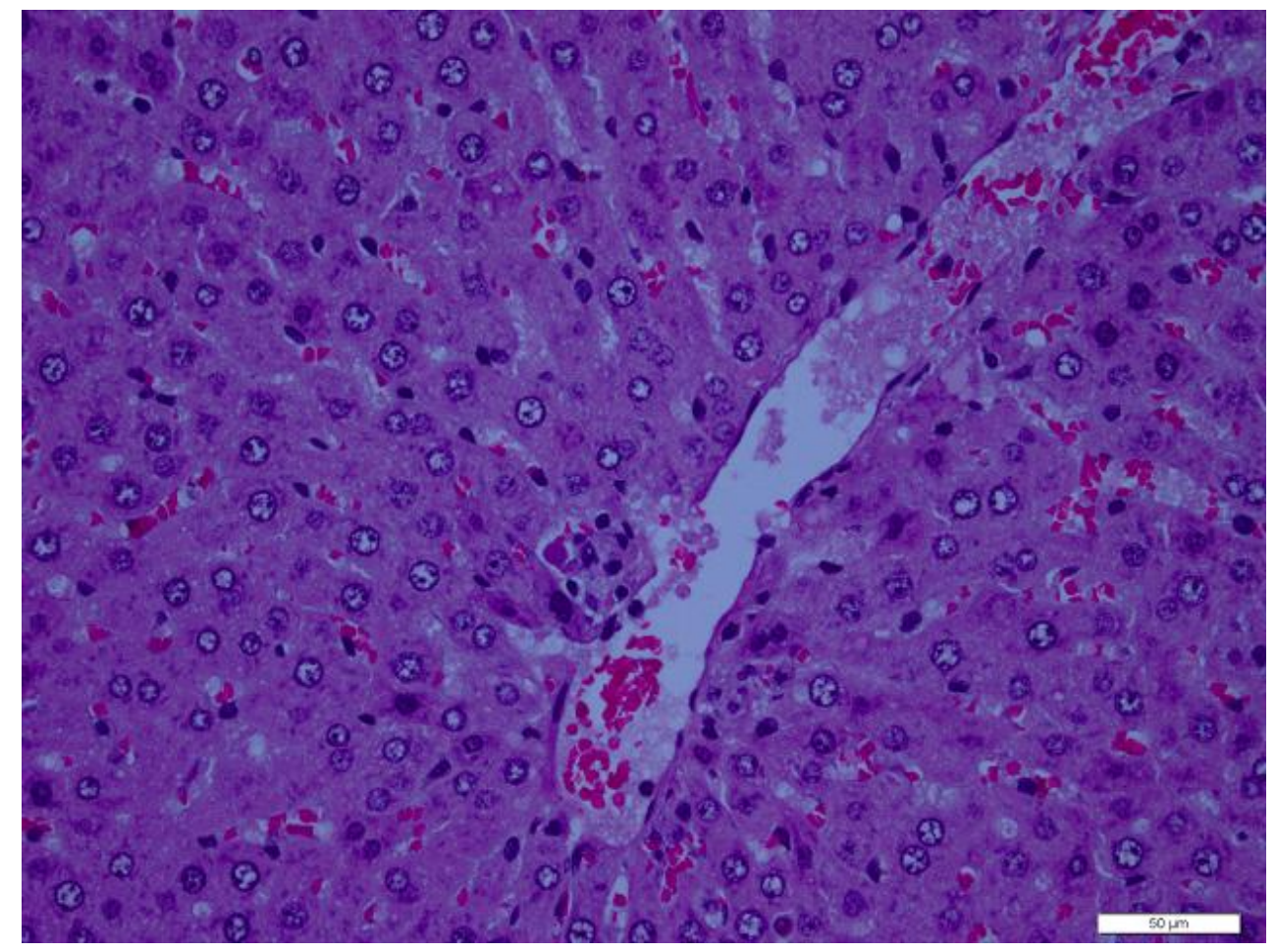

Figure 25 - Histology slide of unheated liver sample (40X)

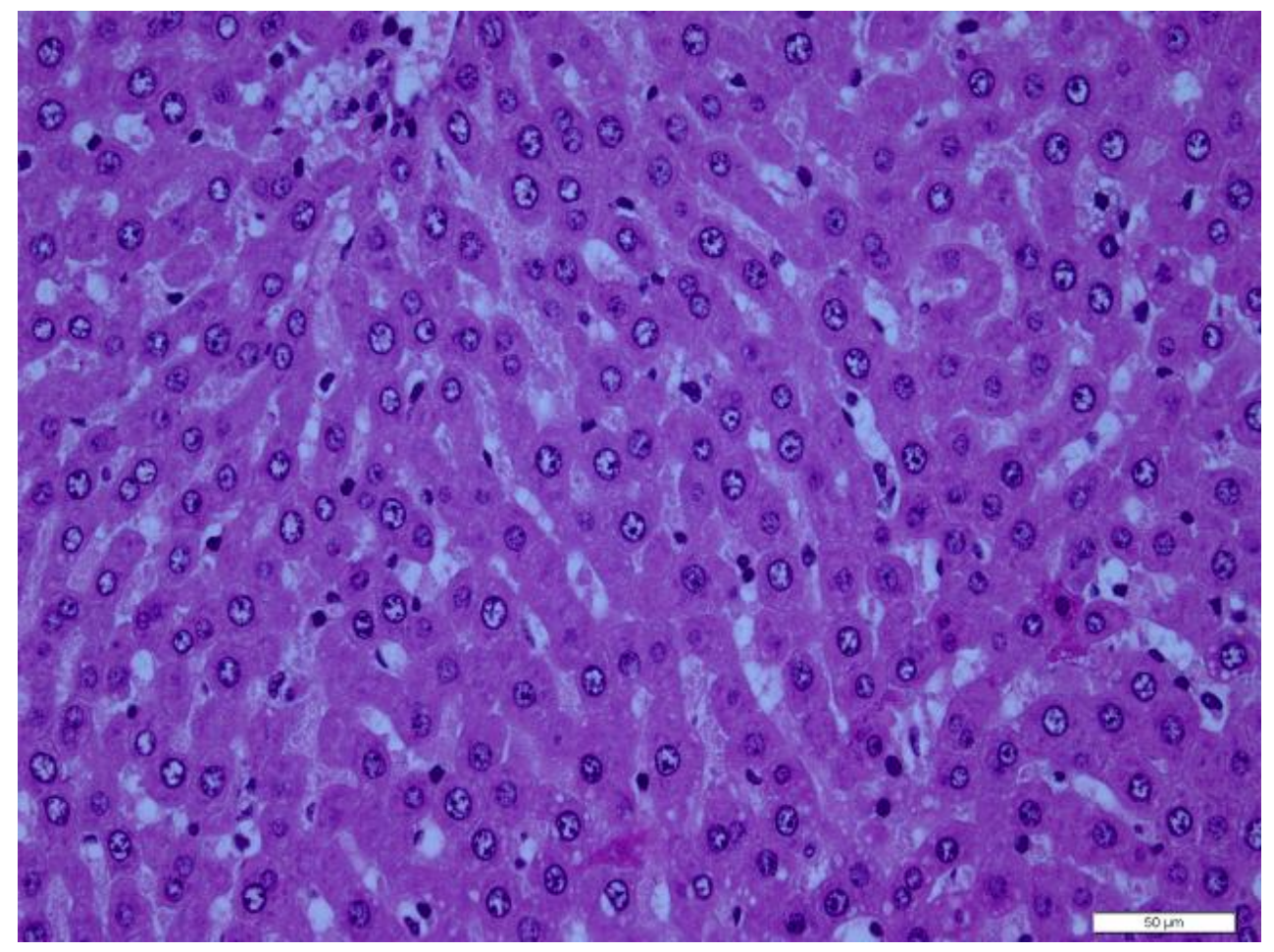

Figure 26 - Histology slide of heated liver sample (40X) 


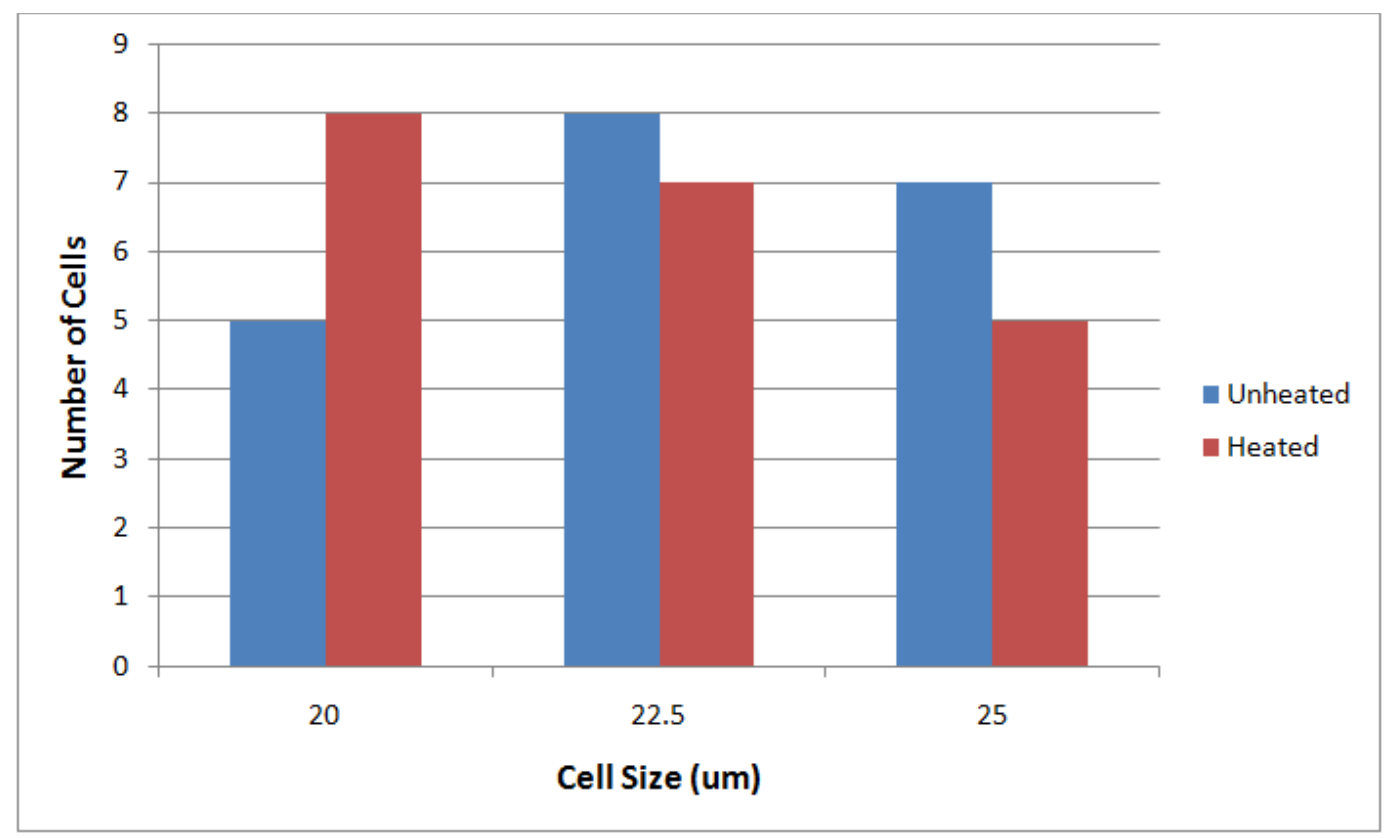

Figure 27 - Histogram of liver cell diameter estimated from histology

\subsection{HIFU Experiments}

A series of HIFU experiments were conducted to assess the extent to which induced damage in tissue was measureable with ultrasound. Liver samples were exposed to a variety of intensities and for durations varying from several seconds to minutes. Changes in average BSC were estimated from regions of the samples designated as corresponding to visible lesions from photographs. In addition, difference images for QUS parameters of ESD and EAC were generated to compare the extent of changes in the lesion region to those in the remainder of the sample. It is important to note that attenuation changes were neglected in this analysis because of the difficultly in estimating a spatially-varying attenuation map from the measurements that were taken. Uniform attenuation changes could not be assumed, as was done in the saline bath experiment, and so all estimates were generated assuming an unheated value of attenuation $(0.65 \mathrm{~dB} / \mathrm{cm} / \mathrm{MHz})$, which was considered to be an important source of error. Table 2 lists the sample exposure conditions for each of the samples shown. 
Table 2 - HIFU exposure parameters

\begin{tabular}{|c|c|c|c|}
\hline \multirow{2}{*}{ Sample \# } & \multicolumn{3}{|c|}{ Exposure Parameters } \\
\cline { 2 - 4 } & Duration (s) & $\begin{array}{c}\text { Peak } \\
\text { Intensity }\left(\mathbf{W} / \text { cm }^{2} \text { ) }\right.\end{array}$ & $\begin{array}{c}\text { B-mode } \\
\text { Visible? }\end{array}$ \\
\hline R3163 & $30 \mathrm{~s}$ & 2500 & Y \\
\hline R3170 & $30 \mathrm{~s}$ & 2500 & $\mathrm{~N}$ \\
\hline R3193 & $10 \mathrm{~s}$ & 1500 & $\mathrm{~N}$ \\
\hline R3410a & $120 \mathrm{~s}$ & 19 & $\mathrm{~N}$ \\
\hline R3410b & $120 \mathrm{~s}$ & 19 & $\mathrm{~N}$ \\
\hline
\end{tabular}

\section{2a Backscatter Coefficient Estimates}

BSC estimates were processed for the same spatial ROIs before and after exposure to HIFU. The ROIs were selected based on photographs of each sample and correspond to the area of visible discoloration due to heating. Because the samples were encased in agar, attenuation through the agar was assumed to be $0.1 \mathrm{~dB} / \mathrm{cm} / \mathrm{MHz}$, where present. Figure 28 shows the average BSC for each individual sample, both before and after exposure to HIFU, excluding the sample where brightening occurred in the B-mode image, resulting in a dramatic change in BSC. Figure 29 shows the average of the BSCs of all liver samples exposed to HIFU, as well as the average of the BSCs of all samples in the saline bath experiment. The slope of the average BSC of heated HIFU samples appeared to closely match the slope of the average BSC of the unheated cases. The slope of the average BSC of the heated saline bath cases was higher, suggesting that the heated HIFU samples may be undercompensated for attenuation. 


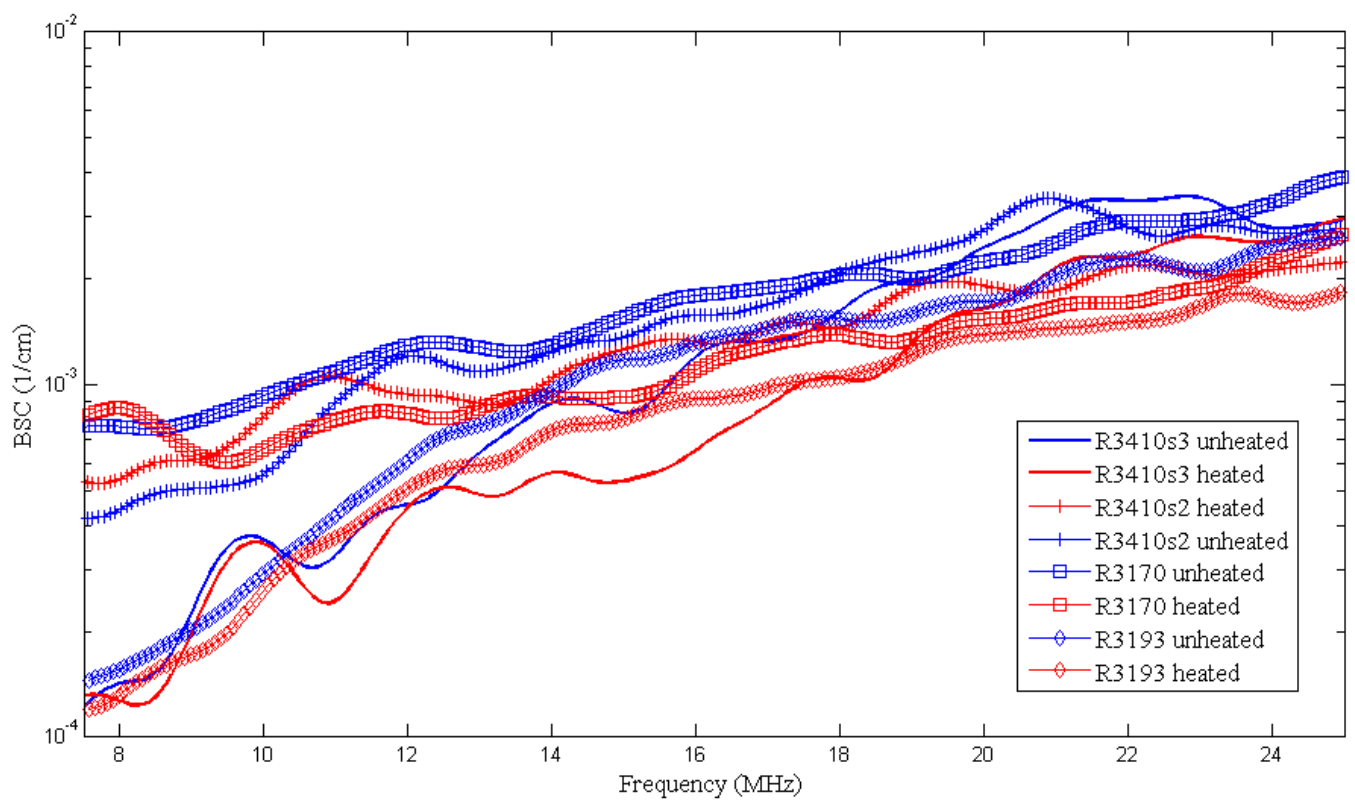

Figure 28 -Backscatter coefficient of rat liver samples exposed to HIFU

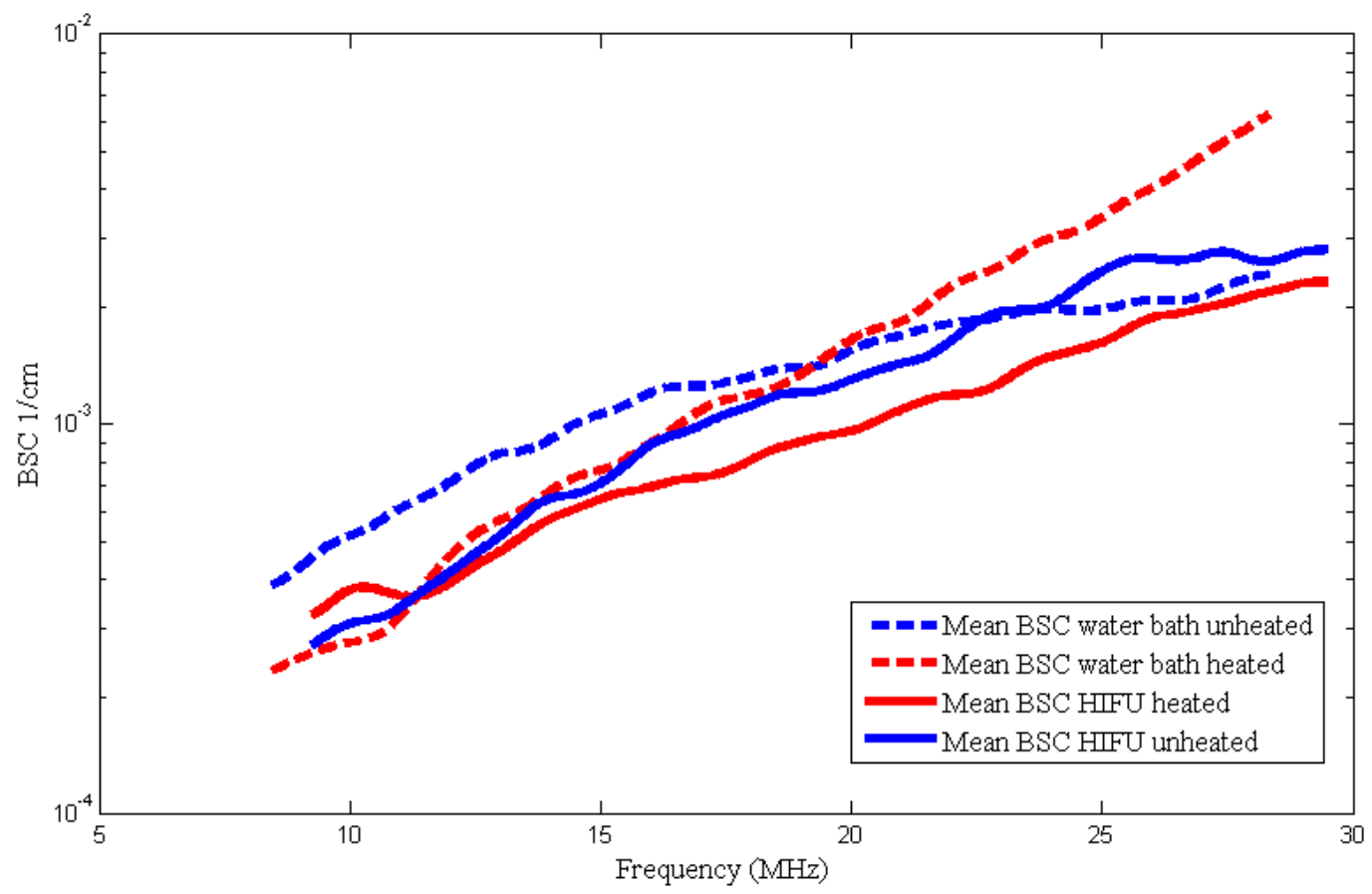

Figure 29 - Average backscatter coefficient of all rat liver samples (thermal HIFU and saline bath) 


\section{2b ESD and EAC}

The QUS parameters of ESD and EAC were evaluated in a similar manner to the saline bath experiments, using three models to develop estimates. Figures 30, 31, and 32 show the mean and variance of estimates for the spherical Gaussian, fluid-filled sphere, and linear models, respectively. ESD was found to increase with heating, while EAC decreased. Scatter plots (figs. 33-35) for each of these form factors do not show the clear separation that was present in the saline bath heating results, suggesting that variation between samples was as large as, or larger than, estimated changes in QUS parameters. Also, unlike the saline bath experiment, the area of effect from HIFU exposure was not uniform, and for a particular ROI, the BSC parameters could consist of contributions from scatterers with a variety of properties. Still, statistically significant differences $(\mathrm{p}<0.05)$ in QUS estimates were found for several sample/parameter combinations according to the results shown in tables 3 and 4.

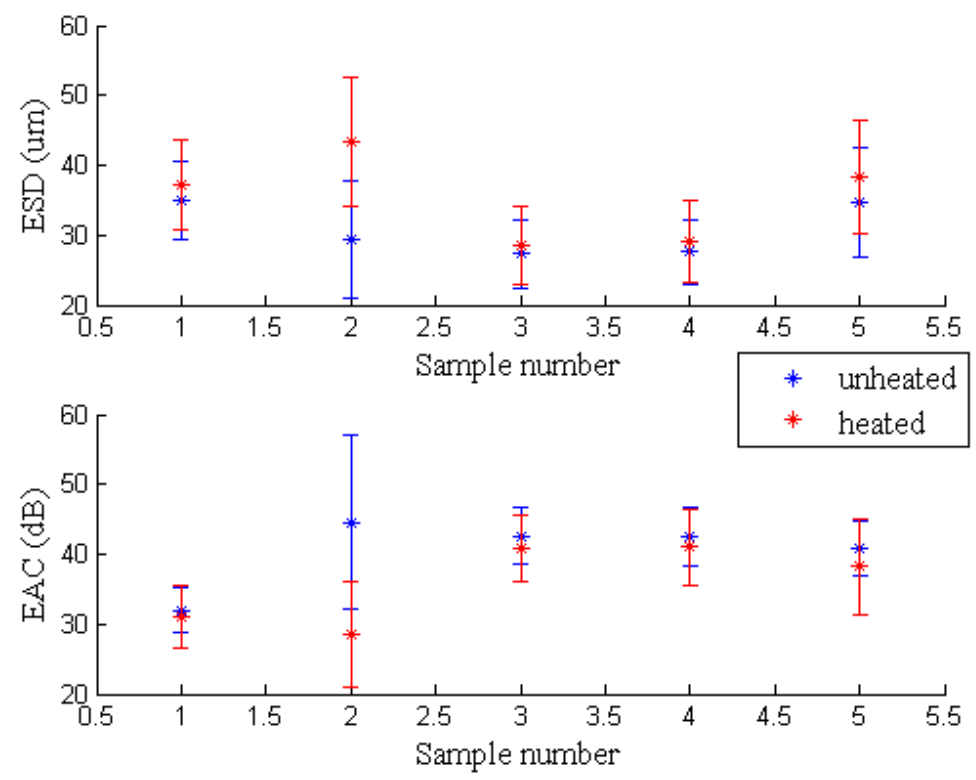

Figure 30 - Mean of QUS parameters (Gaussian form factor) 


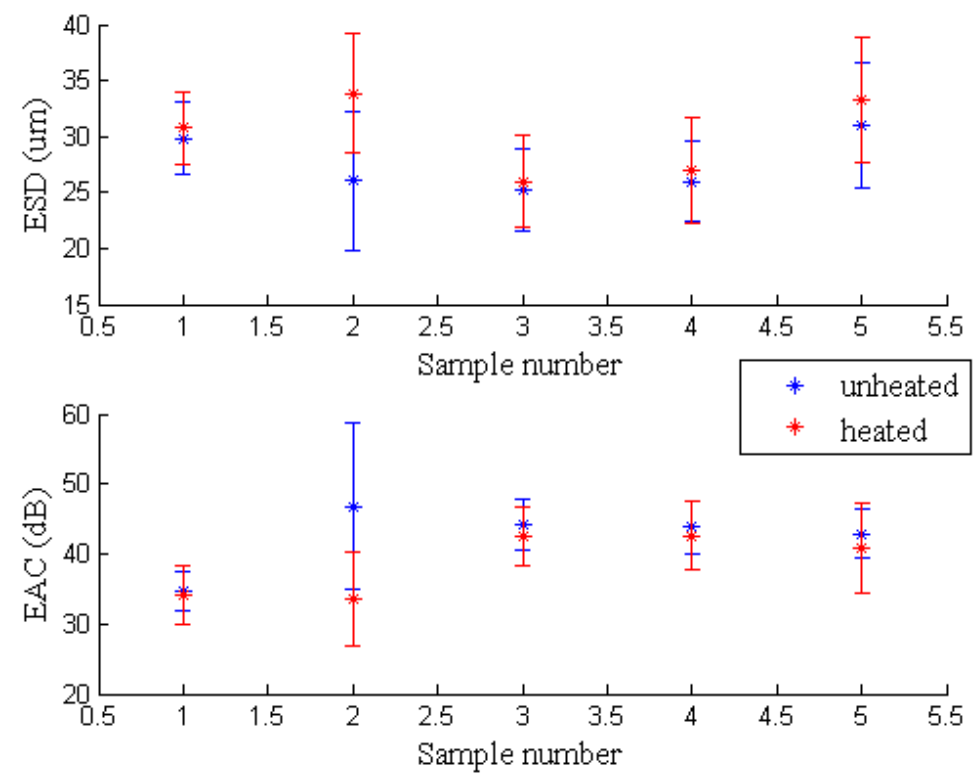

Figure 31 - Mean of QUS parameters (fluid-filled sphere form factor)

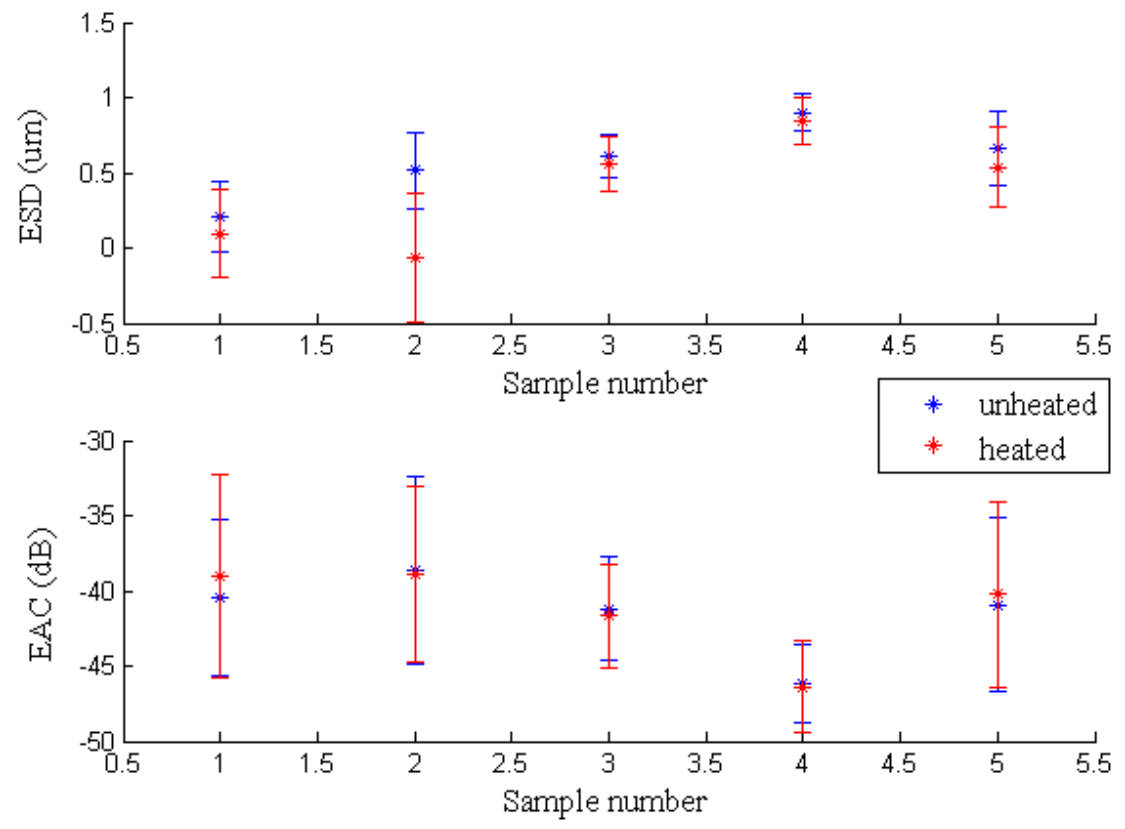

Figure 32 - Mean of QUS parameters (linear model) 


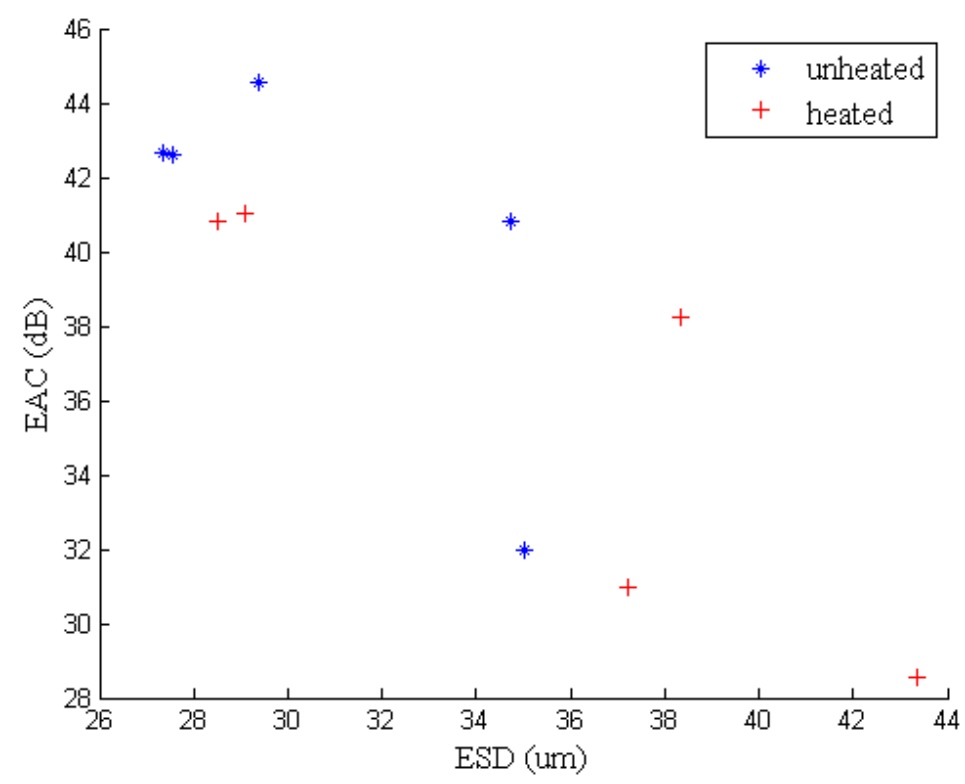

Figure 33 - Scatter plot of QUS parameters (spherical Gaussian model)

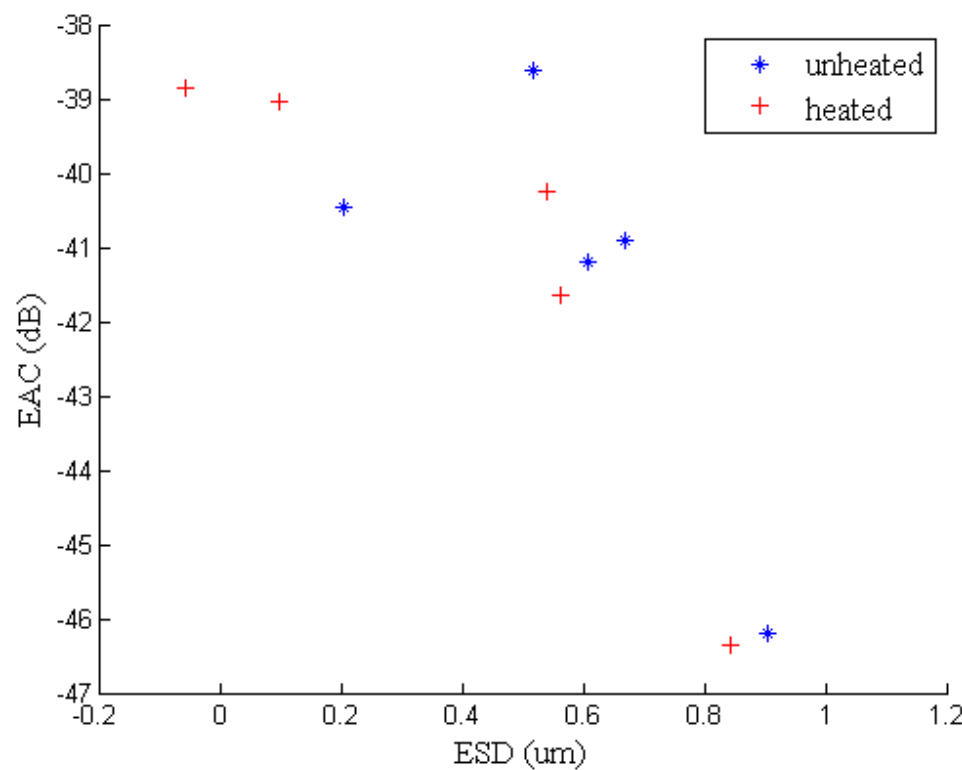

Figure 34 - Scatter plot of QUS parameters (linear model) 


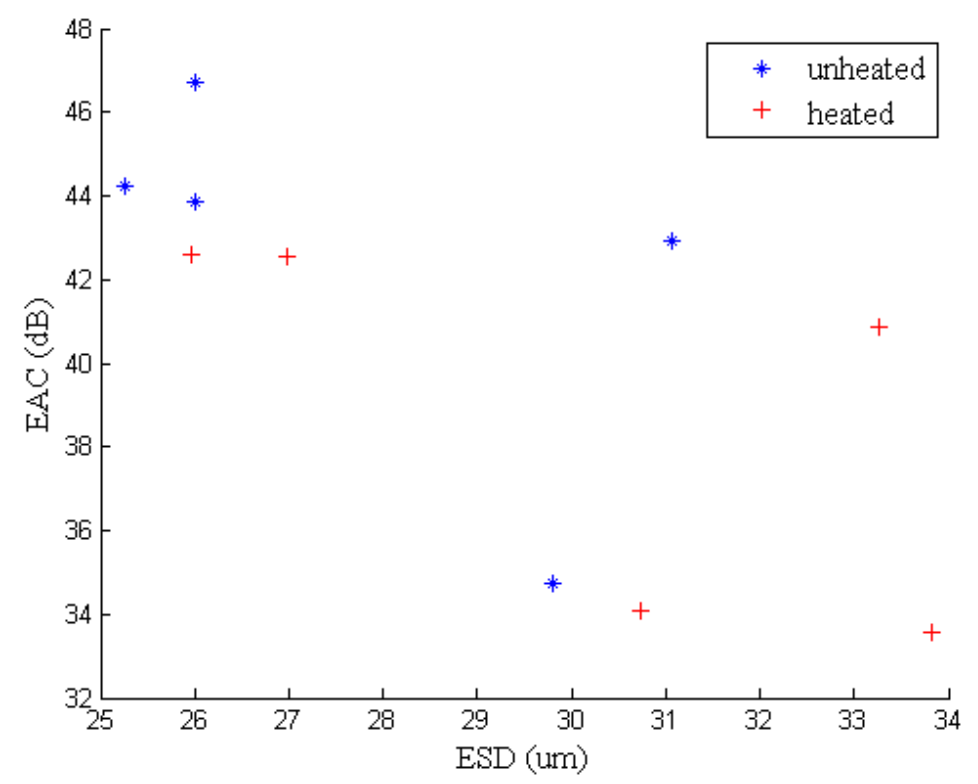

Figure 35 - Scatter plot of QUS parameters (fluid-filled sphere model)

Table 3 - ANOVA for HIFU samples

\begin{tabular}{|c|c|c|c|}
\hline \multirow{2}{*}{ Sample \# } & \multicolumn{3}{|c|}{ p< 0.05 } \\
\cline { 2 - 4 } & $\begin{array}{c}\text { Gaussian } \\
\text { ESD }\end{array}$ & $\begin{array}{c}\text { Fluid-filled } \\
\text { sphere ESD }\end{array}$ & Linear Slope \\
\hline R3163 & Y & Y & Y \\
\hline R3170 & Y & Y & Y \\
\hline R3193 & N & N & N \\
\hline R3410a & N & N & N \\
\hline R3410b & Y & Y & Y \\
\hline
\end{tabular}


Table 4 - ANOVA for HIFU samples

\begin{tabular}{|c|c|c|c|}
\hline \multirow{2}{*}{ Sample \# } & \multicolumn{3}{|c|}{$\mathbf{p}<\mathbf{0 . 0 5}$} \\
\cline { 2 - 4 } & $\begin{array}{c}\text { Gaussian } \\
\text { EAC }\end{array}$ & $\begin{array}{c}\text { Fluid-filled } \\
\text { sphere EAC }\end{array}$ & $\begin{array}{c}\text { Linear } \\
\text { Intercept }\end{array}$ \\
\hline R3163 & Y & Y & Y \\
\hline R3170 & Y & Y & N \\
\hline R3193 & Y & Y & Y \\
\hline R3410a & N & N & N \\
\hline R3410b & Y & Y & N \\
\hline
\end{tabular}

\section{2c Envelope Statistics}

Envelope statistics parameters were generated for HIFU liver data sets, and compared before and after HIFU exposure in ROIs correlated with lesions based on visual inspection of each sample. Both $\mu$ and $k$ parameters were relatively insensitive to changes with heating, as shown in figures 36 and 37 . Statistically significant differences $(\mathrm{p}<0.05)$ between before and after were observed for only 1 of 5 samples for both $\mu$ and $k$.
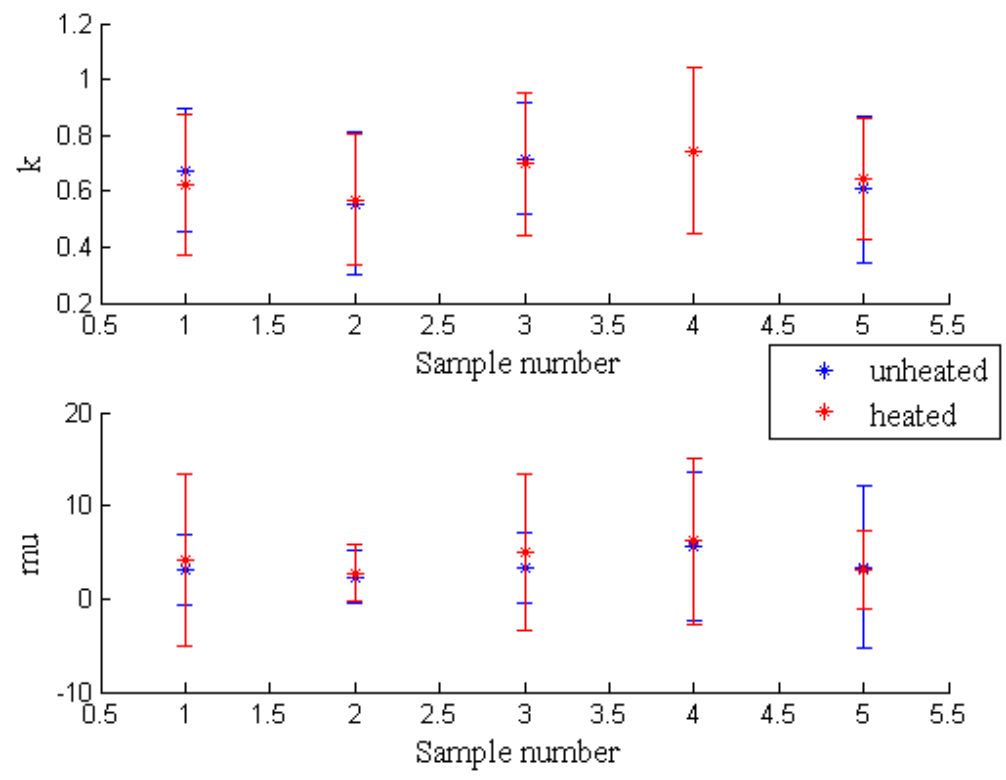

Figure 36 - Mean of envelope statistics parameters 


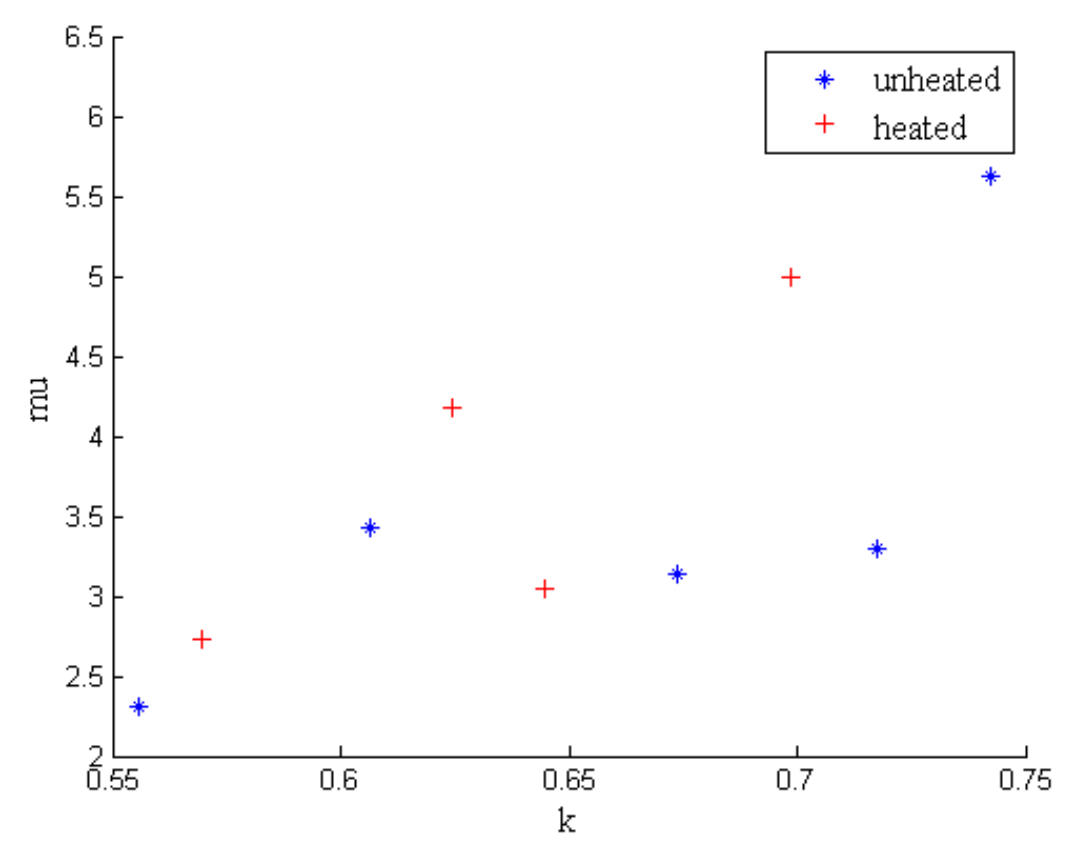

Figure 37 - Scatter plot of QUS envelope statistics parameters

\section{2d QUS Difference Images}

Ultrasound scans were performed on liver samples both before and after exposure to HIFU in the focal region of the HIFU transducer. QUS images were generated for both the before and after cases with overlapping ROIs to show a smoother picture, and difference maps were generated to show changes in QUS parameters with heating, allowing a comparison of changes in the lesion compared to unexposed areas.

It has been suggested [36] that brightening of B-mode images during HIFU exposure may be linked to cavitation activity. In order to maintain as valid as possible a comparison between HIFU exposure and saline bath heating, HIFU exposures and sample handling procedures that avoid such brightening were sought, although this technique did not rule out the presence of cavitation. Table 2 lists the exposures which were found to produce a substantial brightening that was visible on a B-mode scan after the exposure. As expected, QUS images for these samples displayed significant changes with respect to HIFU exposure, as this brightening corresponded to a significant change in estimated scattering properties. Figure 38 shows photographic, B-mode, and QUS images of such a sample. 

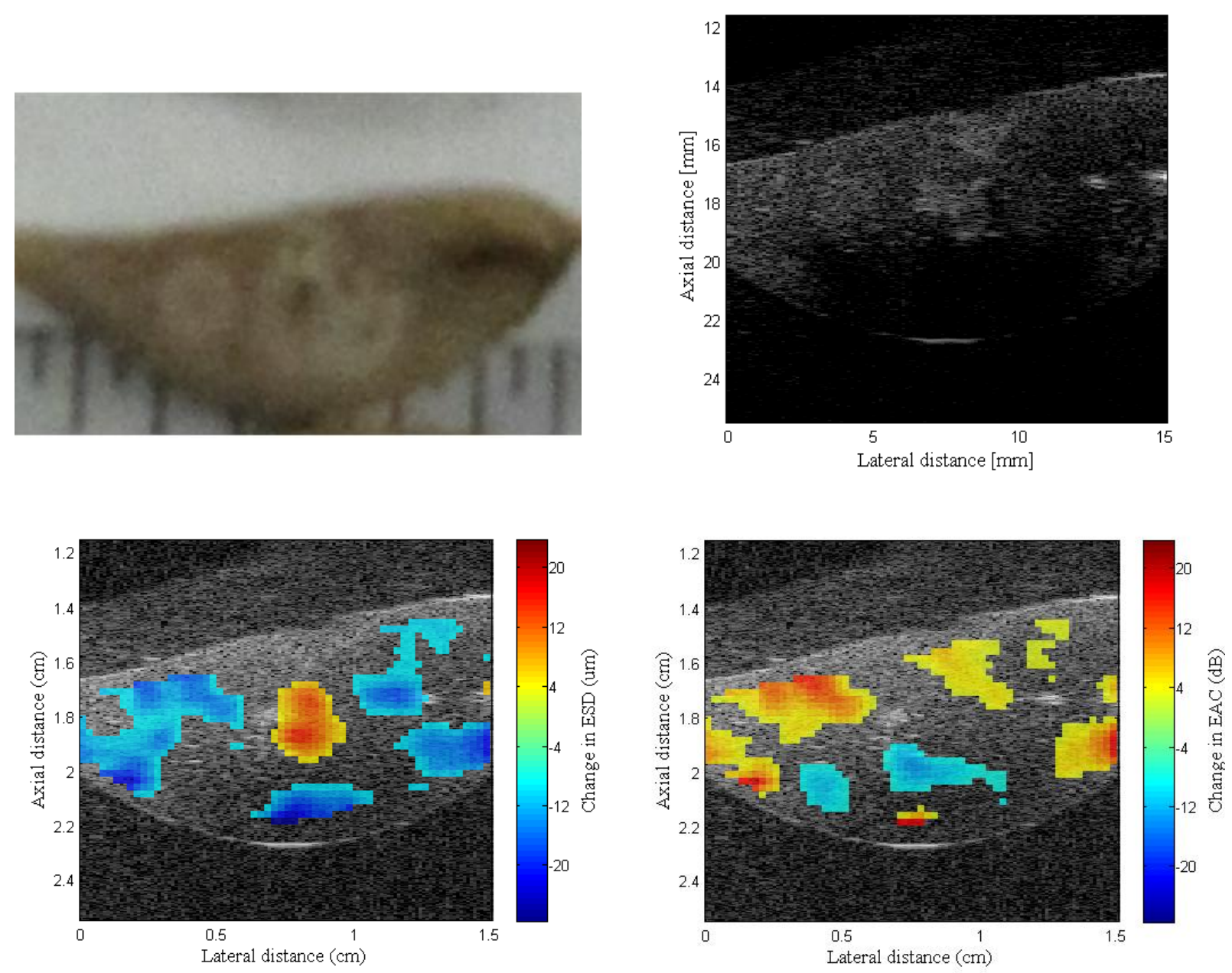

Figure 38 - (Clockwise from top left) photograph, B-mode image, ESD, and EAC images of HIFU rat liver sample

An increase in ESD and decrease in EAC appeared in the QUS images in figure 38. It was noted that the increase in ESD appeared close to the center of the lesion as determined from the photograph, while the decrease in EAC was observed below the center of the lesion. This inconsistency between changes in the saline bath and HIFU experiments may be explained by the potential role of cavitation, by uncompensated changes in attenuation, or by the non-uniform creation of a lesion from HIFU. As can be observed in the sample photograph, a small cavity was formed at the center of the lesion, suggesting the tissue was completely annihilated. In order simulate the saline bath experiment more closely, HIFU exposures were performed which demonstrated no substantial brightening in assessment B-mode images in the area of the visible lesion. The areas of color outside the lesion are due to slight de-correlation between before and after images. 
Figures 39 shows a sample that was exposed under conditions similar to those of the sample of figure 38, but that showed no significant brightening or formation of a cavity. An increase in ESD and decrease in EAC was also observed for this sample. Figure 40 shows a third example exposed under very different conditions (R3140b in table 2) which may have more closely simulated a purely thermal exposure. Again, an increase in ESD and decrease in EAC was observed. Figure 41 shows the measured temperature history for this exposure by a wire thermocouple placed approximately $2 \mathrm{~mm}$ from the center of the focus.
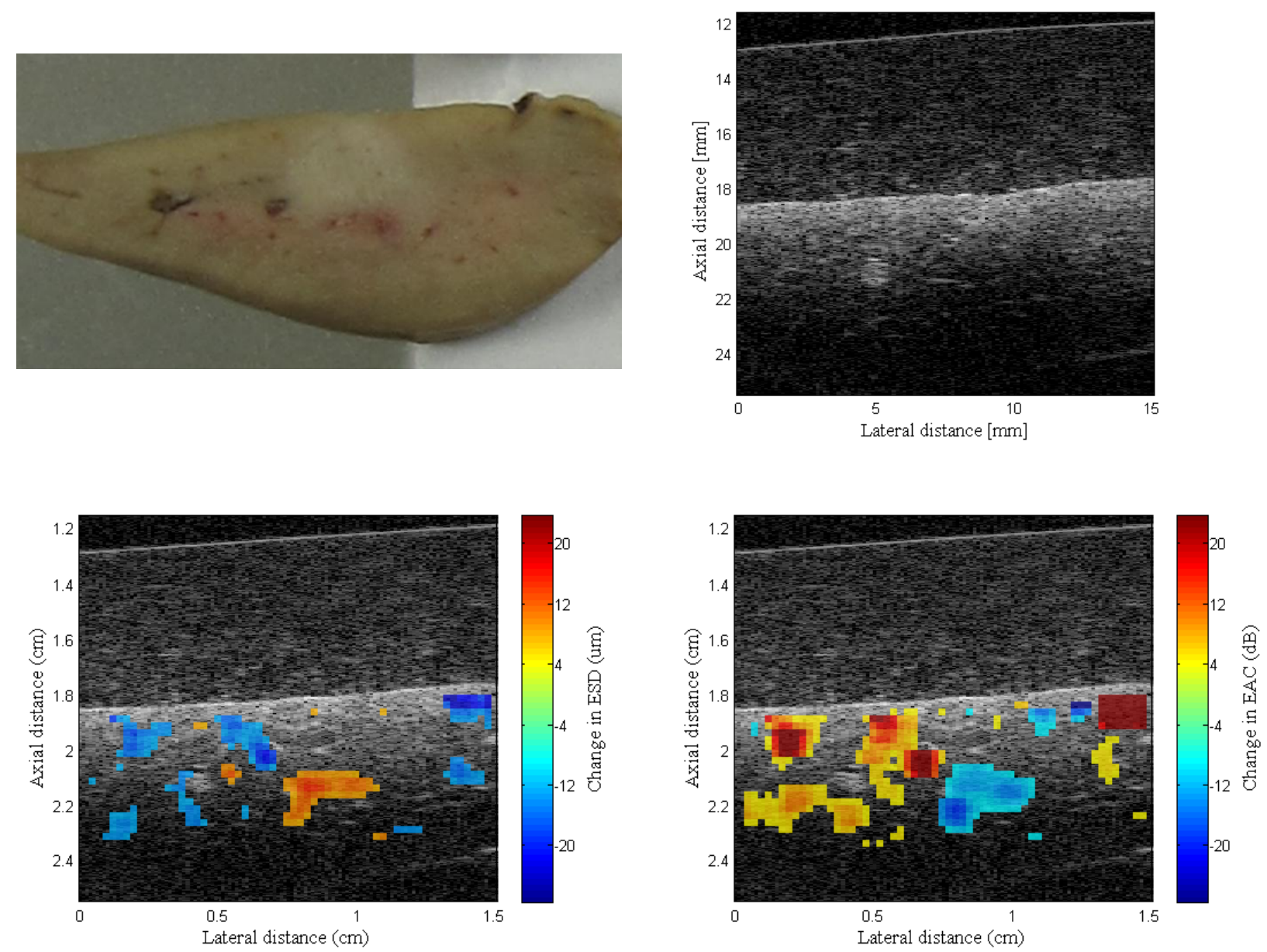

Figure 39 - (Clockwise from top left) photograph, B-mode image, ESD, and EAC images of HIFU rat liver sample 

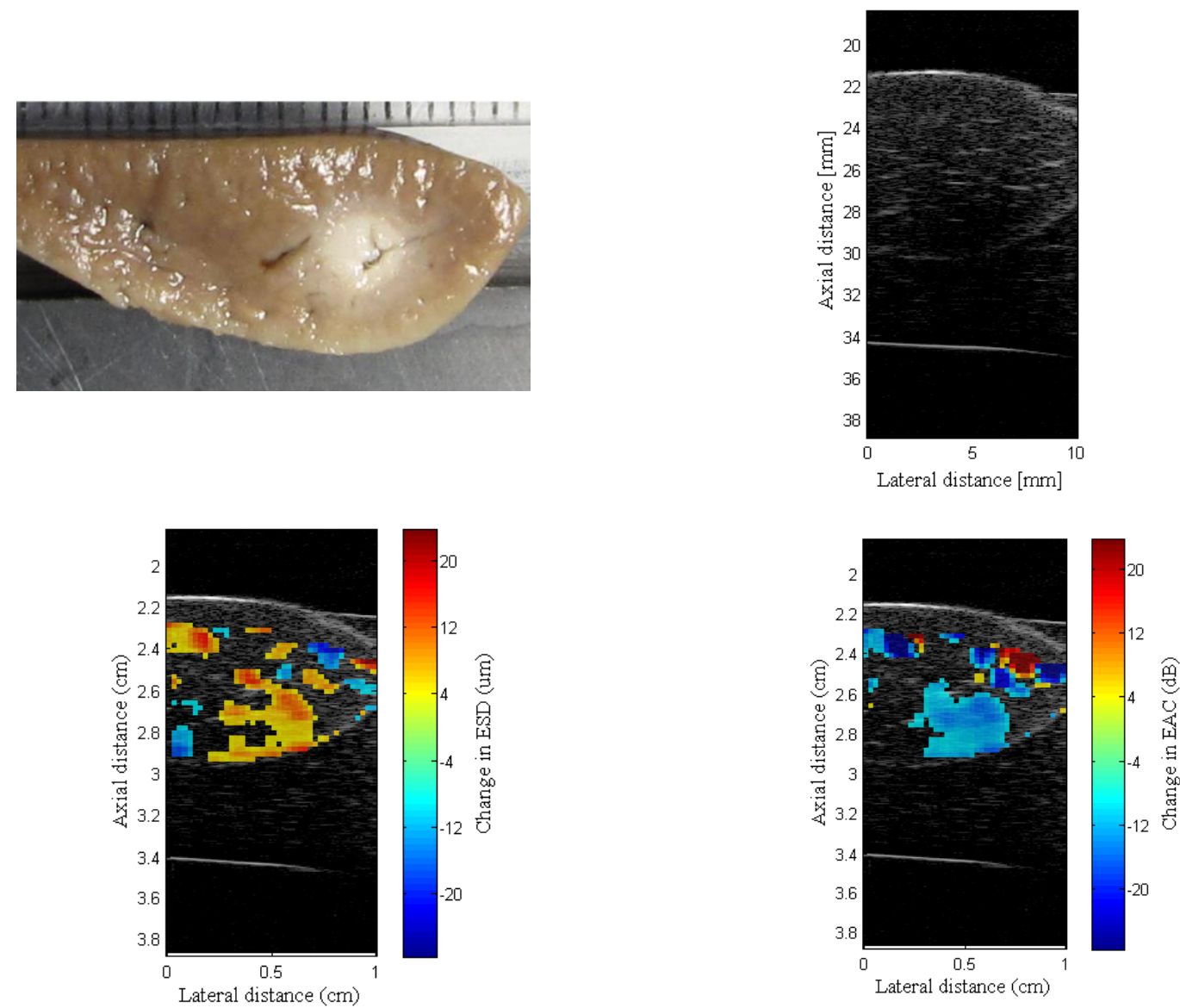

Figure 40 - (Clockwise from top left) photograph, B-mode image, ESD, and EAC images of HIFU rat liver sample 


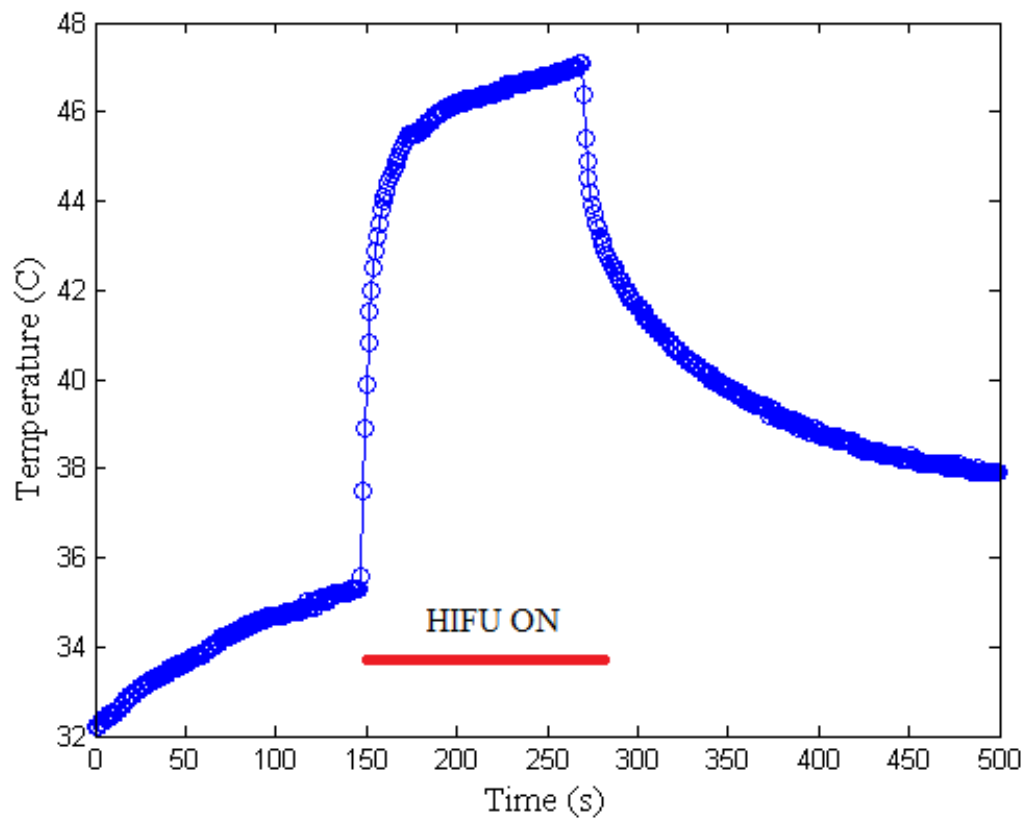

Figure 41 - Temperature history of HIFU exposure

\section{2e Histology Images}

Histological slides were created from liver samples exposed to HIFU. Figure 42 shows a digitized slide image at low magnification (4X) which shows a visible ring of lighter color, corresponding to the location of the circular lesion. At higher magnification (40X), an effect similar to that observed in the histology slides taken from the saline bath heating experiments was observed (sec. 4.1d), where the sinusoids appear dilated. Figure 43 shows an unexposed sample region, which appears darker and to have larger cells than the heated sample region (fig. 44), which shows enlarged sinusoids. Figure 45 shows a histogram of cell size for heated and unheated regions of a liver sample corresponding to figures 44 and 45, respectively. As was the case for the saline bath experiment histology slides, cells diameter was estimated from a print of the histology image, and estimates correspond to the largest dimension of a cell. The average cell diameter for the unheated cells was $22.5 \pm 1.9 \mu \mathrm{m}$, while the average cell diameter for the heated cells was $22.4 \pm 2.4 \mu \mathrm{m}$. The average cell nucleus diameter was $8.8 \pm 1.5 \mu \mathrm{m}$ for unheated cells, and $7.9 \pm 0.9 \mu \mathrm{m}$ for heated cells. 


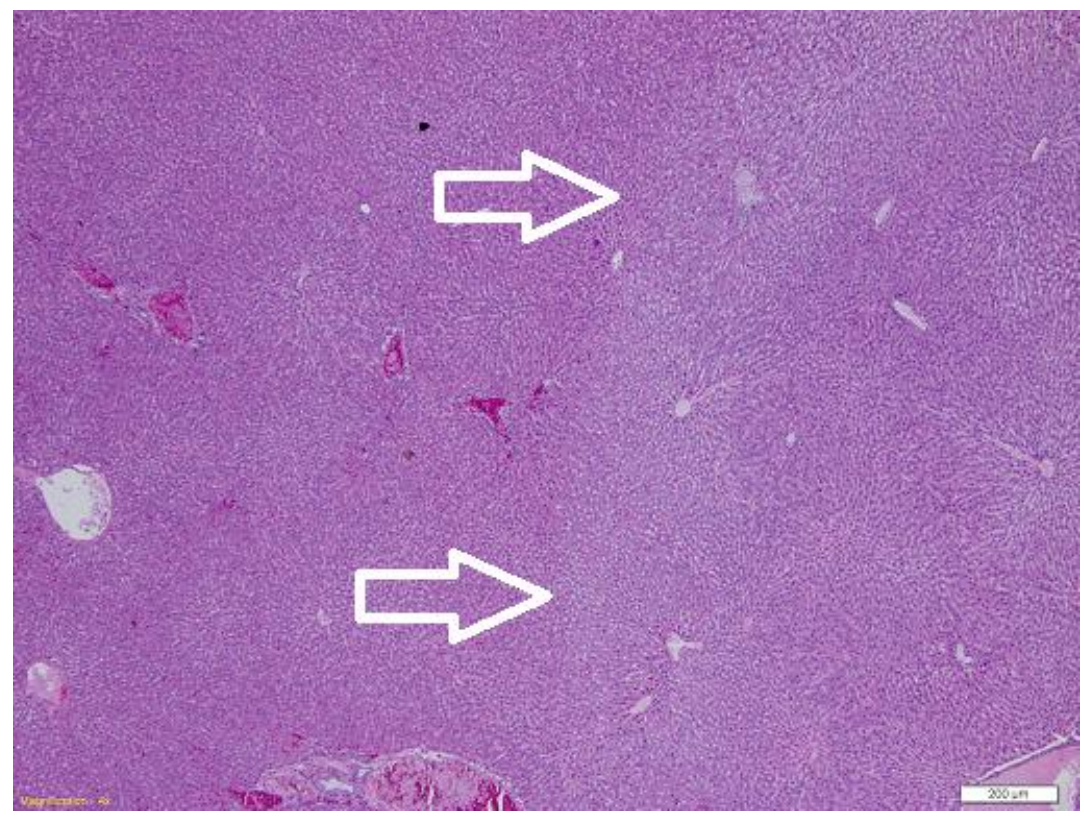

Figure 42 - Histological slide image of HIFU-exposed liver sample (4X, arrows indicate threshold of treated region)

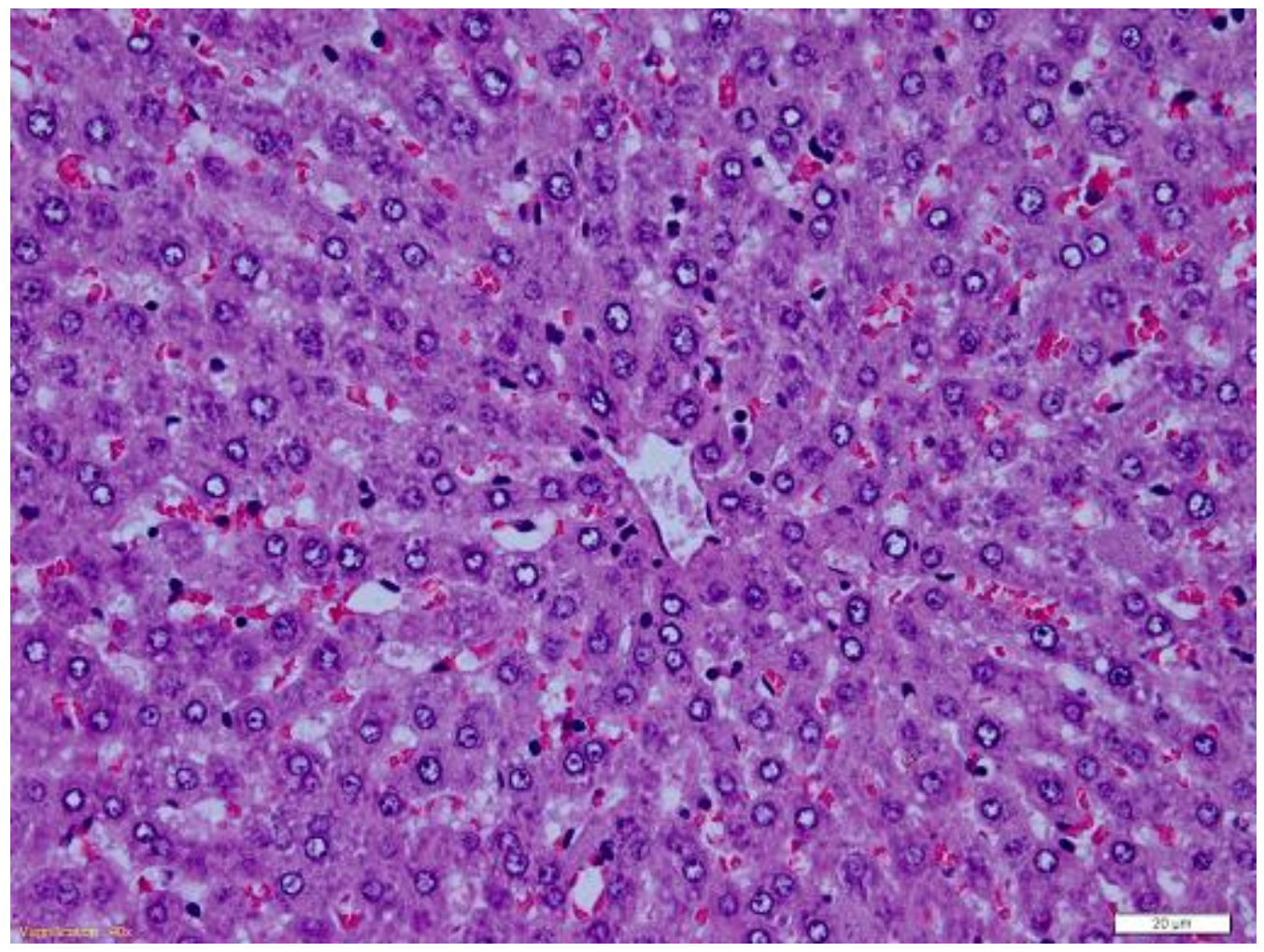

Figure 43 - Histological slide image of HIFU-exposed liver sample (unexposed region, 40X) 


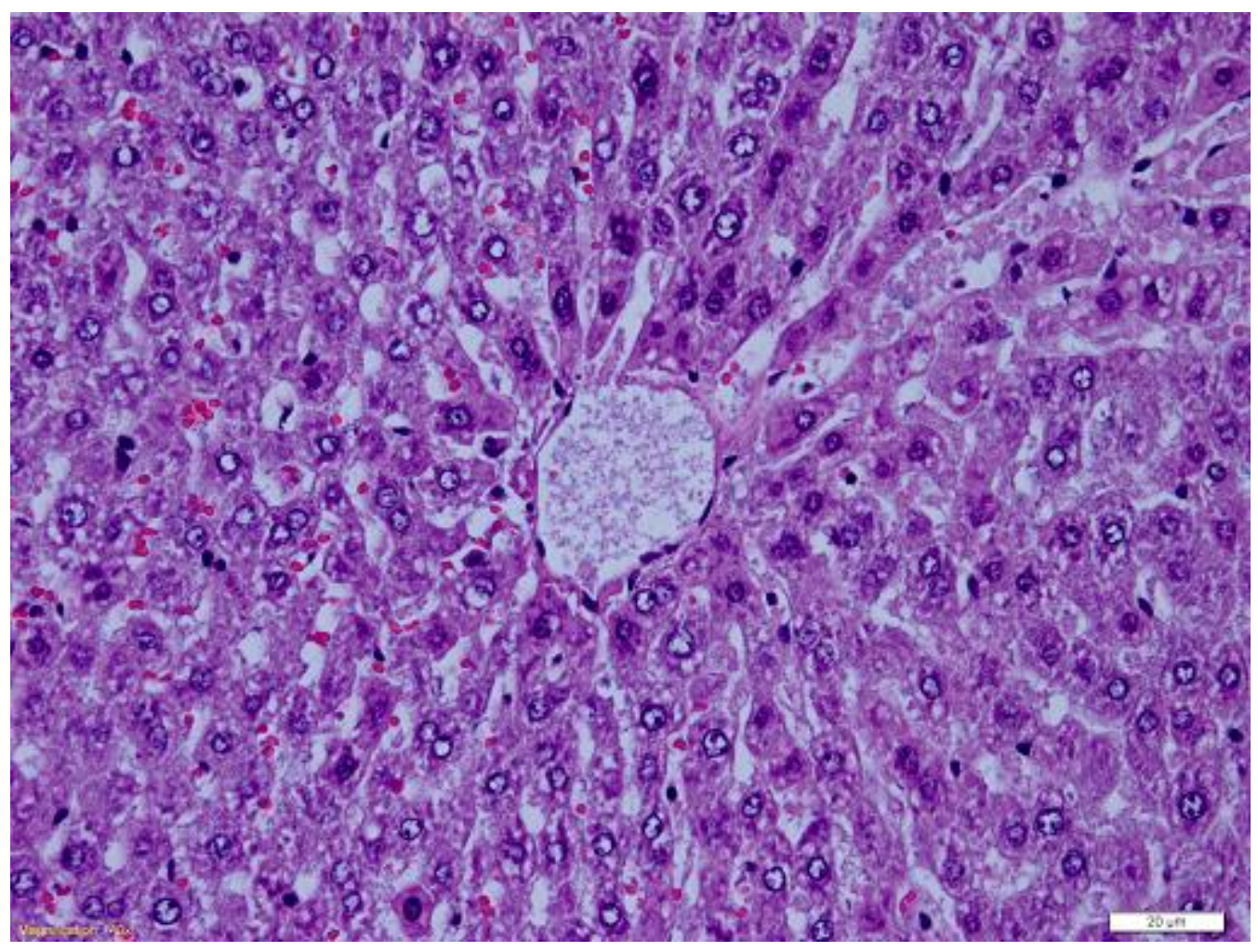

Figure 44 - Histological slide image of HIFU-exposed liver sample (exposed region, 40X)

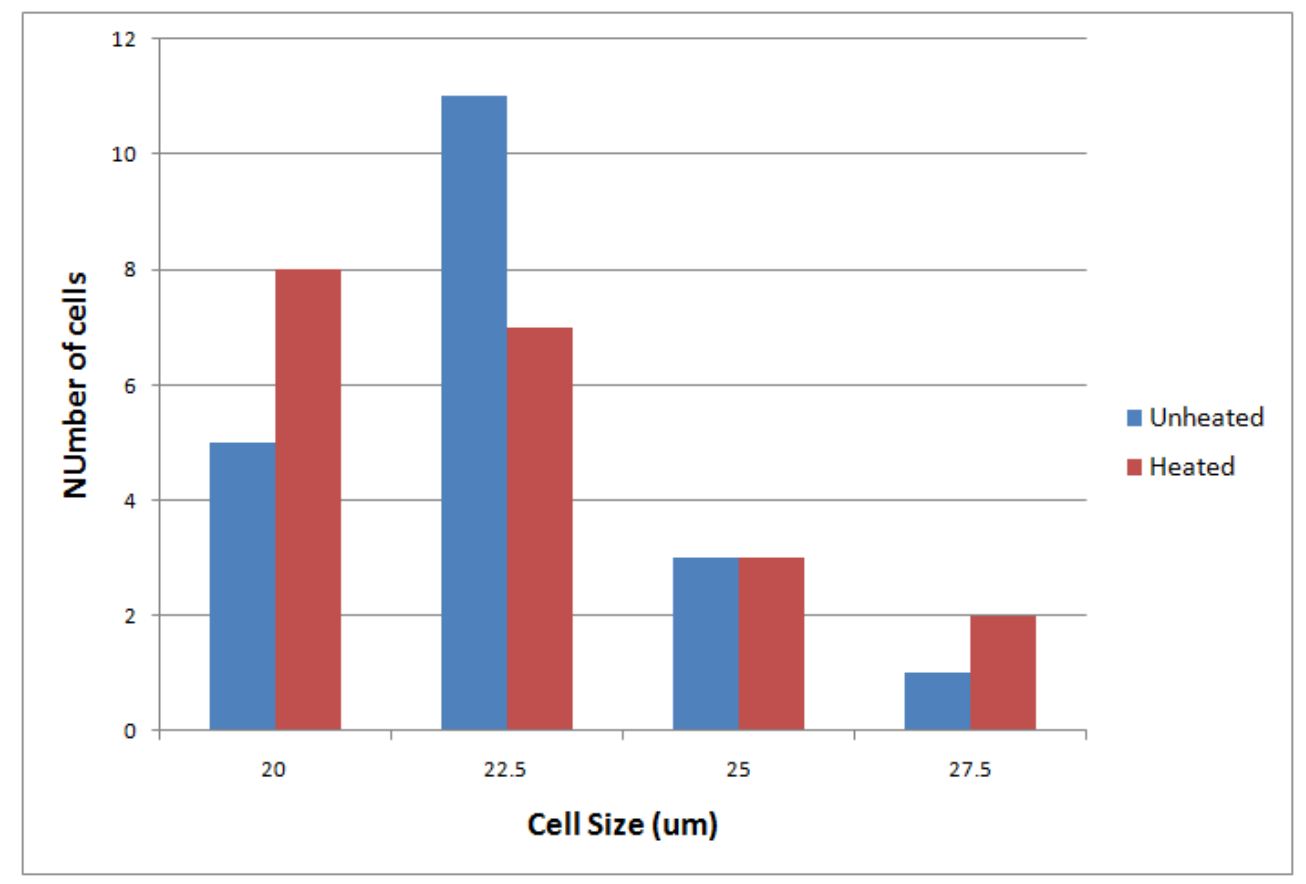

Figure 45 - Histogram of liver cell diameter estimates from histology 


\section{Chapter 5: Conclusions and Future Work}

This work explored the feasibility and practical limitations of using QUS to assess thermal therapy in general, and HIFU therapy in particular. Although changes in scattering and attenuation in liver with heating have been previously examined [12], this work is novel in its use of a higher frequency (20 $\mathrm{MHz}$ ) and model-based QUS estimates. A series of saline bath heating experiments were conducted as an important preliminary step in determining the extent to which changes in liver tissue properties with heating could be measured ultrasonically. The saline bath experiments yielded substantial changes in estimates of sample attenuation with heating, as well as significant and consistent changes in BSCs in liver with heating. While the former has been shown previously and agrees with existing literature, the changes in BSC have not been previously documented. Although macromolecular changes that may contribute to increased absorption and thereby sample attenuation in heated samples could not be verified histologically, scattering models applied to the measured changes in BSCs yielded a decrease in ESD with heating. This decrease was consistent with changes in cell size estimated from histology for the saline bath experiment, where the estimated size of the cells decreased by $2.5 \%$. Estimated size of the cell nuclei increased by $4 \%$, however. Expanded histological analysis will be necessary to understand the changes in cell size that may occur with heating, specifically taking into account different functional regions of the liver.

Although the average change in ESD with heating was consistent with histological evidence, the spherical Gaussian and fluid-filled sphere models did not appear to be a close fit to the averaged BSC estimated in the saline bath experiments. In fact, in both unheated and heated cases a linear model (in logarithmic space) had a lower MSE when compared to the average measured BSCs than both these models. An important future direction of this research will be to explain this discrepancy and to develop a more realistic model for scattering from liver tissue, if needed.

Envelope statistics parameters were considered as a means to discriminate between unheated and heated liver samples. In the saline bath experiment, the $k$ parameter, which is a measure of the ratio of coherent to diffuse scattering, yielded contrast between the heated and unheated samples, while the $\mu$ parameter had no discernable change. Histological evidence does not directly explain these findings, and while the $k$ parameter was found to consistently decrease in heated samples, the differences were not statistically significant within any single sample.

Whereas the saline bath experiments were designed to provide insight into how liver tissue changed with thermal insult, HIFU experiments were conducted to incorporate more realistic conditions with respect to clinical therapy. Several practical challenges, including sample preparation, ultrasound 
exposure duration and intensity, temperature measurement, and lesion registration were overcome that were not present in the saline bath experiment. In contrast to the decreasing ESD estimated in the saline bath experiment with heat, an increase in ESD was estimated in the HIFU heating experiment. Also, the ESD changes did not correlate with histological findings, where the average estimated cell size changed by less than $1 \%$, and estimates of the size of the nuclei decreased by $9 \%$. While the presence of mechanical sources of damage such as cavitation cannot be excluded as a possible explanation, the discrepancy in the results between the HIFU and saline bath experiments appears consistent with undercompensated attenuation in the heated HIFU samples. The EAC in particular was consistently lower in ROIs directly beneath the lesion as determined by photograph evidence, suggesting that a significant uncompensated attenuation in the path to that area may be present.

Uncompensated attenuation changes explain the discrepancy between the ESD estimates in the HIFU and saline bath experiments, as undercompensated ROIs will lead to larger ESD estimates. Furthermore, lesions formed by HIFU are not uniform in their effect, unlike in the saline bath experiments, and scatterer property estimates from ROIs correlated spatially with the HIFU lesions may consist of scatterers with a variety of properties. Aside from reducing the contrast between affected and unaffected regions, the ability to observe statistically significant differences would also be lessened. Although scatter plots indicated that changes in ESD and EAC with HIFU heating were small compared to sample variation, several sample/model pairs had statistically significant differences between before and after estimates. Envelope statistics parameters were insensitive to changes with HIFU heating, as only one sample was observed to have a statistically significant difference in either parameter.

Of the three models (spherical Gaussian, fluid-filled sphere, linear) and five QUS parameters (ESD, EAC, attenuation, $\mu, k$ ), attenuation and ESD appeared to be the most reliable indicators of thermal damage. The spherical Gaussian and fluid-filled sphere models produced very similar estimates in the saline bath experiment, resulting in a clear separation between heated and unheated regions, while the linear model had the lowest MSE between measured and theoretical BSC curves. Changes in estimated attenuation coefficient with heating in saline were substantial. ESD had lower variance in general than EAC, which had large variance for the heated samples. Also, ESD and EAC appeared to be somewhat correlated, suggesting the ESD alone may be a useful indicator of heating. Envelope statistics parameters were not as sensitive to tissue damage from heating as ESD and EAC in either the saline bath or HIFU experiment.

The effects on the estimated power spectrum due to changes in scattering and attenuation appeared to be opposite in the HIFU experiment, resulting in measurable changes in ESD and EAC only 
at locations in depth where attenuation changes dominated. The estimates in these areas did not agree with the saline bath experiments or with histological findings. For this reason, a robust and reliable way to estimate attenuation changes in tissue independently of ESD and EAC would provide an important complement to existing QUS methods.

The findings of this work reflected positively on the potential of QUS for assessment of HIFU and thermal therapy. Changes in attenuation coefficient, BSC, and related QUS parameters have demonstrated the sensitivity of QUS to changes in liver tissue as the result of heating. An important experimental challenge highlighted by this work was to generate damage in liver ultrasonically that could be considered purely thermal in nature, thereby making a comparison of the saline bath heating and HIFU experimental results more meaningful. Two additional challenges were to measure changes in attenuation with HIFU exposure, which could be quite large and have a significant impact on QUS estimates, and to explain the apparent discrepancy between measured and modeled BSC for liver. Continuing work in QUS for HIFU assessment will focus on these challenges, along with developing a more systematic way to define and measure the exposure dose for HIFU and thermal therapy in tissues. 


\section{References}

[1] J. W. Barnard, W. J. Fry, F. J. Fry, and R. F. Krumins, "Effects of high intensity ultrasound on the central nervous system of the cat," The Journal of Comparative Neurology, vol. 103, no. 3, pp. 459-484, December 1955.

[2] J. E. Kennedy, G. R. ter Haar, and D. Cranston, "High intensity focused ultrasound: surgery of the future?" British Journal of Radiology, vol. 76, pp. 590-599, 2003.

[3] F.J. Fry, and L.K. Johnson, "Tumor irradiation with intense ultrasound," Ultrasound in Medicine and Biology, vol. 4, no. 4, pp 337-341, October 1978.

[4] G. ter Haar, I. Rivens, L. Chen, and S. Riddler, "High intensity focused ultrasound for the treatment of rat tumors," Physics in Medicine and Biology, vol. 36, no. 11, 1495-1501, November 1991.

[5] L. Chen, I. Rivens, G. ter Haar, S. Riddler, C. R. Hill, and J. P .M . Bensted, "Histological changes in rat liver tumours treated with high-intensity focused ultrasound," Ultrasound in Medicine and Biology, vol. 19, no. 1, pp 67-74, 1993.

[6] L. Chen, G. ter Haar, C.R. Hill, S. A. Eccles, and G. Box, "Treatment of implanted liver tumors with focused ultrasound," Ultrasound in Medicine and Biology, vol. 24, no. 9, pp 1475-1488, December 1998.

[7] C.R. Hill, I. Rivens, M. G. Vaughan, and G. R. ter Haar, "Lesion development in focused ultrasound surgery: a general model," Ultrasound in Medicine and Biology, vol. 20, no. 3, pp 259-269, 1994.

[8] C. Damianou and K. Hynynen, "The effect of various physical parameters on the size and shape of necrosed tissue volume during ultrasound surgery," Journal of the Acoustical Society of America, vol. 95, pp. 1641-1649, March 1994.

[9] K. Hynynen, "The threshold for thermally significant cavitation in dog's thigh muscle in vivo," Ultrasound in Medicine and Biology, vol. 17, no. 2, pp 157-169, 1991.

[10] N. McDannold, M. Moss, R. Killiany, D. L. Rosene, R. L. King, F.A. Jolesz, and K. Hynynen, "MRI-guided focused ultrasound surgery in the brain: Tests in a primate model," Magnetic Resonance in Medicine, vol. 49, pp. 1188-1191, 2003.

[11] K. Hynynen, O. Pomeroy, D. N. Smith, P. E. Huber, N. J. McDannold, J. Kettenbach, J. Baum, S. Singer, and F. A. Jolesz, "MR imaging-guided focused ultrasound surgery of fibroadenomas in the breast: a feasibility study," Radiology, vol. 219, pp. 176-185, April 2001.

[12] J.C. Bamber and R.C. Hill, "Ultrasonic attenuation and propagation speed in mammalian tissues as a function of temperature," Ultrasound in Medicine and Biology, vol. 5, pp. 149-157, 1979.

[13] M.R. Gertner, B.D. Wilson, and M.D. Sherar, "Ultrasound properties of liver tissue during heating," Ultrasound in Medicine and Biology, vol. 23, no. 9, pp 1395-1403, 1997.

[14] C. Simon, P. VanBaren, and E. Ebbini, "Two-dimensional temperature estimation using diagnostic ultrasound," IEEE Transactions of Ultrasonics, Ferroelectronics, and Frequency Control, vol. 45, no. 4, pp. 1088-1099, July1998.

[15] R. Arthur, J. Trobaugh, W. Straube, and E. Moros, "Temperature dependence of ultrasonic backscattered energy in motion-compensated images," IEEE Transactions of Ultrasonics, Ferroelectronics, and Frequency Control, vol. 52, no. 10, pp. 1644-1652, October 2005. 
[16] N.L. Bush, I. Rivens, G. R. ter Haar, and J. C. Bamber, "Acoustic properties of lesions generated with an ultrasound therapy system," Ultrasound in Medicine and Biology, vol. 19, no. 9, pp. 789801, 1993.

[17] Xinliang Zheng and Shahram Vaezy, "An acoustic backscatter-based method for localization of lesions induced by high-intensity focused ultrasound," Ultrasound in Medicine and Biology, vol. 36, no. 4, pp. 610-622, 2010.

[18] J. Ophir, I. Cespedes, H. Ponnekanti, Y. Yazdi, and X. Li, "Elastrography: A quantitative method for imaging the elasticity of biological tissues," Ultrasonic Imaging, vol. 13, pp 111-134, 1991.

[19] F. L. Lizzi, M. Ostromogilsky, E. J. Feleppa, M. C. Rorke, and M. M. Yaremko, "Relationship of ultrasonic spectral parameters to features of tissue microstructure," IEEE Transactions of Ultrasonics, Ferroelectronics, and Frequency Control, vol. 33, no. 3, pp. 319-329, May 1987.

[20] E. J. Feleppa, A. Kalisz, J. B. Sokil-Melgar, F. L. Lizzi, T. Liu, A. L. Rosado, M. C. Shao, W. R. Fair, Y. Wang, M. S. Cookson, V. E. Reuter, and W. D. W. Heston, "Typing of prostate tissue by ultrasonic spectrum analysis," IEEE Transactions of Ultrasonics, Ferroelectronics, and Frequency Control, vol. 43, no. 4, July 1996.

[21] M. F. Insana, R. F Wagner, D. G. Brown, and T. J. Hall, "Describing small-scale structure in random media using pulse-echo ultrasound," Journal of the Acoustical Society of America, vol. 87, no. 1, pp. 179-192, January 1990.

[22] M. L. Oelze, W. D. O'Brien, J. P. Blue, and J. F. Zachary, "Differentiation and characterization of rat mammary fibroadenomas and 4T1 mouse carcinomas using quantitative ultrasound imaging," IEEE Transactions on Medical Imaging, vol. 23, no. 6, June 2004.

[23] M. L. Oelze and J. F. Zachary, "Examination of cancer in mouse models using high-frequency quantitative ultrasound," Ultrasound in Medicine and Biology, vol. 32, no. 11, pp. 1639-1648, November 2006.

[24] J. Mamou, A. Coron, M. Hata, J. Machi, E. Yanagihara, P. Laugier, and E. J. Feleppa, “ThreeDimensional High-Frequency Characterization of Cancerous Lymph Nodes," Ultrasound in Medicine and Biology, vol. 36, no. 3, pp. 361-375, March 2010.

[25] J. Mamou, A. Coron, M. L. Oelze, E. Saegusa-Beecroft, M. Hata, P. Lee, J. Machi, E. Yanagihara, P. Laugier, and E. J. Feleppa, "Three-dimensional high-frequency backscatter and envelope quantification of cancerous human lymph nodes," Ultrasound in Medicine and Biology, vol. 37, no. 3, pp. 345-357, March 2011.

[26] F. L. Lizzi, M. Astor, T. Liu, C. Deng, D. J. Coleman, and R. H. Silverman, "Ultrasonic spectrum analysis for tissue assays and therapy evaluation," International Journal of Imaging Systems and Technology, vol. 8, no. 1, pp. 3-10, 1997.

[27] G. J. Czarnota, M. C. Kolios, J. Abraham, M. Portnoy, F. P. Ottensmeyer, J. W. Hunt, and M. D. Sherar, "Ultrasound imaging of apoptosis: high resolution non-invasive monitoring of programmed cell death in vitro, in situ and in vivo," British Journal of Cancer, vol. 81, no. 3, pp. 520-527, October 1999.

[28] E. H. Wissler, "Pennes' 1948 paper revisited," Journal of Applied Physiology, vol. 85, no. 1, pp. 35-41, July 1998.

[29] M. L. Oelze, J. F. Zachary, and W. D. O'Brien, "Characterization of tissue microstructure using ultrasonic backscatter: theory and technique for optimization using a Gaussian form factor," Journal of the Acoustical Society of America, vol. 112, pp. 1202-1211, September 2002. 
[30] V. C. Anderson, "Sound scattering from a fluid sphere," Journal of the Acoustical Society of America, vol. 22, no. 4, pp. 426-431, July 1950.

[31] D. P. Hruska and M. L. Oelze, "Improved parameter estimates based on the homodyned K distribution," IEEE Transactions of Ultrasonics, Ferroelectronics, and Frequency Control, vol 56, no. 11, pp. 2471-2481, November 2009.

[32] T. A. Bigelow, M. L. Oelze, and W. D. O’Brien, "Estimation of total attenuation and scatterer size from backscattered ultrasound waveforms," Journal of the Acoustical Society of America, vol. 117, no. 3, pp. 1431-1439, March 2005.

[33] T.A. Bigelow, B. L. McFarlin, W. D. O’Brien, and M. L. Oelze, "In-vivo ultrasonic attenuation slope estimates for detecting cervical ripening in rats: Preliminary results," Journal of the Acoustical Society of America, vol. 123, no. 3, pp. 1794-1800, March 2008.

[34] K. J. Parker, "Ultrasonic attenuation and absorption in liver tissue," Ultrasound in Medicine and Biology, vol. 9, no. 4, pp. 363-369, July 1983.

[35] S. A. Goss, L.A. Frizzell, and F. Dunn, "Ultrasonic absorption and attenuation in mammalian tissues," Ultrasound in Medicine and Biology, vol. 5, no. 2, pp. 181-186, 1979.

[36] B. A. Rabkin, V. Zderic, and S. Vaezy, "Hyperecho in ultrasound images of HIFU therapy: Involvement of cavitation," Ultrasound in Medicine and Biology, vol. 31, no. 7, pp. 947-956, July 2005 . 